\title{
Follow the Majority? How Voters Coordinate Electoral Support to Secure Club Goods*
}

\author{
Dominik Duell ${ }^{\dagger}$
}

August 31, 2020

\begin{abstract}
Voters often favor candidates who benefit them individually but may coordinate their support with their social group on other candidates in exchange for policies targeting their group. In a laboratory experiment, I induce group identities to investigate the behavior of voters facing such trade-offs. I find that groups with low within heterogeneity often secure the club good from a candidate who is also individually beneficial to a majority of the group. In more heterogeneous groups, coordination on that candidate often fails and while the group still receives club goods, it is from a candidate whose policies are otherwise individually costly to most of the group. The results highlight the role strategic considerations play in the formation of group-based electoral coalitions.
\end{abstract}

${ }^{*}$ The research presented in this paper was supported by NSF Grant \#SES-1124265 and the Wilf Family Department of Politics at New York University. Support through the ANR - Labex IAST is also gratefully acknowledged. I thank Dimitri Landa, Joshua Tucker, Catherine Hafer, Rebecca Morton, Eric Dickson, Tiberiu Dragu, Sönke Ehret, Sandy Gordon, Karine van der Straeten, Patrick Egan, Robert Johns, Catherine de Vries, Andre Blais, Christine Stedtnitz, Adam Peresman, and Rachel Schutte for their thoughtful comments and suggestions and Lorenz Kurrek for research assistance.

${ }^{\dagger}$ University of Essex, dominik.duell@essex.ac.uk 


\section{Introduction}

Group identities matter in elections and often lead to an electorate divided along group lines (Michelitch, 2015; Eifert, Miguel and Posner, 2010; Huddy, 2001). Intuitively, the mechanism by which individuals with the same group identity align their behavior is rooted in shared preferences or

other group-related considerations, such as concern for group status or group conformity (Akerlof and Kranton, 2000; Dickson and Scheve, 2006). The exact target of joint group action (that is, which candidate the group chooses to support collectively) however, varies significantly with context, such as the distribution of policy preferences within the group or the nature of competition among candidates who offer group-targeted benefits - i.e., club goods - in exchange for the group's electoral support.

The relationship between politicians and voters in many societies is frequently characterized as clientelistic, a trade of beneficial policies for electoral support (Kitschelt and Wilkinson, 2007); examples can be found from New Haven, Connecticut (Dahl, 2005) to Zambia (Posner, 2005). Such policies that exclusively benefit non-universal social groups are a standard feature of politics, even if their "purchase" in Western democracies is not acknowledged by stakeholders as explicitly as it is in patronage systems. Voters reward incumbents for targeted policies (Harding, 2015; Weghorst and Lindberg, 2013; Carlson, 2015) and politicians strategically engage in providing group-targeted, excludable benefits to particular social groups to create and sustain reciprocal relationships (Gottlieb et al., 2019) if possible (Ichino and Nathan, 2013).

In this paper, I provide a theoretical account of exactly how voters coordinate with other members of their social group to secure group-targeted benefits. I show how they trade off their preferences over policies benefiting them at the individual-level against group-level benefits from a club good. In particular, I model the relationship between within-group heterogeneity in policy preferences and the groups' ability to coordinate on one candidate to secure a group-targeted benefit. I then implement a laboratory experiment where subjects in the role of voters of one social group favor a particular candidate given the benefits they would receive individually but may together coordinate their electoral support on any of the candidates in exchange for benefits from a club good they would receive as a member of a particular group. Experimental treatments pinpoint which electoral alternative draws members of one social group with variation in within-group heterogene- 
ity in preferences over individual-level benefits or priming of the individual-level vs group-targeted benefits offered by candidates.

The baseline treatment operationalizes the main dimension of political conflict as a distribution of income with a larger social group comprising mostly wealthy members; a smaller social group that is mostly poor; and two candidates, one proposing redistribution of income and the other a status-quo distribution. The group-targeted benefit is represented by a club good that the social group can secure for all of its members but only if the group helped one of the candidates win and represents a majority of supporters of the winning candidate. ${ }^{1}$ To mimic group identities, I induce minimal groups that have been shown to trigger relevant effects of group categorization in the laboratory (i.e., awareness of group membership and formation of identity-contingent beliefs about others' behavior). ${ }^{2}$

The experiment confirms the models' prediction that when within-group heterogeneity is low, voters mostly coordinate on the equilibrium where they vote for a candidate who benefits most members of the group individually while securing the group-targeted benefit from the winning candidate. Such equilibrium behavior is observationally equivalent to following a group-majoritarian decision heuristic - vote for the candidate whose platform is individually beneficial to most members of the group - providing a clear coordination target. Interestingly, in more heterogeneous groups, for which voting for this candidate is not an equilibrium, I find behavior observationally equivalent to group-majoritarian coordination as well. To a larger extent, more heterogeneous groups converge on a candidate who imposes individual-level costs on most members of the group but delivers the club good; behavior that is congruent with equilibrium play in the model of electoral competition. Experimentally priming the individual-level benefit voters may derive from choosing between candidates makes coordination on that equilibrium candidate less likely, driven by those group members who would incur a cost in reduced individual-level benefits from switching to equilibrium play. Finally, I provide evidence that groups featuring higher within-heterogeneity converge to the

\footnotetext{
${ }^{1}$ The exact distribution of income in this experiment is not meant to be descriptive of a particular real-world instantiation but to create conflicting preferences over individual-level and group-targeted benefits.

${ }^{2}$ Throughout this paper I discuss a group identity that requires individual's subjective awareness of group membership based on a shared trait but may not rise to the level of being a social identity. The latter goes beyond awareness of membership and demands the individual attach value and emotional significance to their membership (Tajfel, 1981). It is debatable whether I am able to induce a social identity with its complex cognitive and emotional underpinnings in the laboratory, but the treatment clearly succeeds in creating a group identity (See manipulation checks presented in Section 4: Experimental design).
} 
equilibrium candidate who is individually costly to most members of the group because of voters' awareness of what their fellow group members might do and how other groups behave. This finding demonstrates the existence of a strong strategic rationale behind the formation of group-based electoral coalitions.

By delineating mechanisms by which voters coordinate on a specific candidate, this study makes three contributions: it adds to the political behavior literature that investigates group identities as political identities (for an overview see Huddy (2013)) and highlights the role strategic considerations play in the formation of electoral coalitions; it extends the literature on ethnic politics investigating the mechanisms behind census voting (Chandra, 2004; Posner, 2005; Eifert, Miguel and Posner, 2010); and it provides a micro-foundation for the beliefs-relevant effects of social group membership, going beyond the seminal framework of Akerlof and Kranton (2000) for conceptualizing group identity. ${ }^{3}$

\section{Group-targeted benefits and electoral coalition formation}

In elections where group identities matter, their influence on individuals' preferences is the most commonly considered mechanism explaining individuals' group-contingent decision-making. Voters give more weight to group-related concerns than in elections without group divisions, where this shift is attributed to general warm-glow in-group favoritism (Andreoni, 1989; Chen and Li, 2009), emotional gains from conforming to group norms (Goette, Huffman and Meier, 2006; Bernhard, Fehr and Fischbacher, 2006; Suhay, 2015), group status (Shayo, 2009; Klor and Shayo, 2010), or acting in conformity with fellow group members (Bernheim, 1994; Hogg, 1996), but also to emotional losses from failing to concede to peer pressure (White, Laird and Allen, 2014). Akerlof and Kranton (2000) prominently introduced such group-driven preferences into models of individual decision-making as an additive term in agents' utility function.

All these effects on vote choice from membership in a social group provide rationales to do what is "best" for one's group or to vote in conformity with one's group. However, groups are often heterogeneous, inter-group competition is complex, and voting for the group's best interest or following

\footnotetext{
${ }^{3}$ Whereas studies employing that framework conceive of group identity as an added term in voters' utility function, by capturing group-related concerns - such as group norms and group status - group identity in the analysis below emerges contingently, affecting individual behavior through a conditional coordination mechanism.
} 
a group norm is not always obvious. Group decision-making, where outcomes have consequences with respect to individual- and group-targeted benefits, presents a coordination problem (Bornstein, 2003). Faced with that problem, individuals as group members intuitively may align their vote choice with what fellow group members are most likely to do. In the context of ethnic politics, Chandra (2004) articulates an important argument that provides a starting point for guiding our thinking about how identities may serve as group coordination devices: we see coordination of vote choice along ethnic lines when co-ethnics represent a large enough electoral coalition to help the candidate win; the candidate, then, provides group-targeted benefits. ${ }^{4}$ The question still arises: On which candidates do voters of the same group coordinate? Do they choose a co-ethnic candidate; but what if such a candidate is not running? Do they pick a candidate who offers club goods; but how do they chose when there are several candidates campaigning on group-targeted benefits? We may expect group members in such circumstances to coordinate by aligning with and supporting the apparent policy preferences of the majority of group members; they would assess those preferences by considering which alternative would give most group members a higher utility. The definition of group-majoritarian coordination follows accordingly: groups coordinate on the electoral alternative that is preferred by most members of that group.

When one electoral alternative is preferred by a majority of group members, most members receive a higher individual-level benefit from supporting that candidate over another. Take, for example, a group whose membership mostly comprises wealthy individuals but also includes some poor individuals. According to group-majoritarian coordination, all members of the group should vote against income redistribution regardless of whether the individual voter is poor. Such behavior is observationally equivalent to the frequently noted phenomena where low-income groups vote for conservative parties when salient social group membership blurs the relationship between income and preferences for redistribution (Roemer, 1998; Scheve and Stasavage, 2006). A vote for a wealthpreserving status quo policy against redistribution would generate higher individual-level benefits for a majority of group members. ${ }^{5}$

Group-majoritarian coordination imposes cost on the fewer poorer members of the group when

\footnotetext{
${ }^{4}$ Among others, there is evidence of coordination driven voting choices in Argentina (Stokes, 2005), South Africa (Ferree, 2006), and Malawi (Ferree and Horowitz, 2010).

${ }^{5}$ In this example, income is just one individual-level characteristics determining the kind of polices that are beneficial to the individual; depending on context, there are many others.
} 
forming an electoral coalition. Is there a strategic rationale that could justify a group attempt to coordinate on the electoral alternative that leads to a loss in individual-level benefits for most members of the of group? Consider the following mechanism: suppose that most members of a society with two social groups would benefit from income redistribution (the median income is below the mean income), but most members of a larger social group are disproportionately wealthy, and those in a smaller, minority social group are mostly poor. When that larger social group is rather heterogeneous in preferences over individual-level benefits or when campaign appeals prime the necessity for a group to coordinate their vote for securing group-targeted benefits, members' awareness of the preferences of their social group as a group - the awareness of the diversity of preferences over the individual-level benefit within their social group - as well as the awareness of the preferences of the poorer minority social group may increase. Subsequently, members of the larger social group will be more aware of the fact that the poorer minority social group prefers redistribution. ${ }^{6}$ At this point, given that the minority social group together with the poorer members of the larger social group may represent an electoral majority of votes in this society, the politician who offers a higher level of redistribution becomes a viable candidate to win the election. The wealthier members of the larger social group may now realize the appeal of voting for more redistribution, since voting jointly with other members of their social group allows them to at least secure the club good for their group. The definition of equilibrium coordination follows accordingly: groups support the electoral alternative that may not be individually beneficial to most group members but secures group-targeted benefits.

Before I describe results from an experiment that tests which coordination mechanism is prevalent, it is necessary to verify that the behavior underlying both mechanism is rationalizable by characterizing equilibrium play in a model of electoral competition.

\section{A simple model of electoral competition}

Electoral competition is modeled in a complete information environment and I provide equilibrium predictions accordingly. Consider a society of $N=5$ voters where voter $i$ is characterized by two

\footnotetext{
${ }^{6}$ In fact, choices by members of the minority social group in the experimental data indicate the prevalence of the belief that voting for the wealth-preserving candidate is, indeed, not worth the attempt. They almost unanimously vote for the redistributive candidate independent of particular income and treatment (See Section 5).
} 
distinct attributes. The first attribute is her level of income $\omega_{i}$ distributed according to $F\left(\omega_{i}\right)$. The second attribute is a binary group identity attribute, which, given $N$ odd, induces a division of voters into two groups, $\{M I, M J\}$, where $M J$ is the larger (majority) social group $\left(N_{\mathrm{MJ}}=3\right)$ and $M I$ is the smaller (minority) social group $\left(N_{\mathrm{MI}}=2\right)$.

The political competition is a majority-voting contest between two candidates, $C=\{P, R\}$. Candidate P's platform is to provide a public good, which voters value at $V$, at the cost of a tax $\tau$ to finance it. Candidate $R$ is the anti-redistribution candidate whose platform is to maintain the existing income levels without redistributive public good provision. Both candidates offer grouptargeted benefits to the group, $M J$ or $M I$, which most strongly supports them electorally. ${ }^{7}$ Voter $i$ chooses which of the candidates to vote for, $a_{i} \in A_{i}=\{P, R\}$.

Voter $i$ 's utility has two components. One component, denoted $U_{i}^{C}$, is the individual-level benefit dependent on $\omega_{i}$ and induced by which candidate wins the election:

$$
U_{i}^{C}= \begin{cases}\omega_{i}(1-\tau)+V & \text { if } P \text { wins } \\ \omega_{i} & \text { if } R \text { wins }\end{cases}
$$

The second component is the utility derived from the group-targeted benefit, denoted $I$, which depends on whether agent $i$ is a member of the social group, $M I$ or $M J$, that represents an electoral majority of voters who supported the winning candidate. Formally,

$$
I= \begin{cases}\mathcal{I} & \text { if } \quad n_{\mathrm{MJ}}>n_{\mathrm{MI}} \text { and } i \in \mathrm{MJ} \\ & n_{\mathrm{MI}}>n_{\mathrm{MJ}} \text { and } i \in \mathrm{MI} \\ \frac{1}{2} \mathcal{I} & \text { if } \quad n_{\mathrm{MJ}}=n_{\mathrm{MI}} \\ 0 & \text { otherwise, }\end{cases}
$$

where $n_{\mathrm{MJ}}$ is the number of voters in $M J$ who voted for the winning candidate, $n_{\mathrm{MI}}$ is the number of voters in the $M I$ who voted for the winning candidate, and $\mathcal{I}>0$. Voter $i$ 's utility is thus, given as $u_{i}=U_{i}^{C}+I$.

The fact that only the latter requires enough voters sharing the same group identity attribute to vote for the same candidate to receive the benefit differentiates individual-level and group-targeted benefit. Voters receive the individual-level benefit provided in the platform of the winning candidate

\footnotetext{
${ }^{7}$ I assume throughout that candidates are committed to implementing their respective platforms if elected and abstract away from the reasons they might have for running. The game that is analyzed is not one of strategic candidate entry as modelled, among others, in Gordon, Huber and Landa (2007); Ashworth and Shotts (2014).
} 
no matter who elected that candidate; a poor voter, for example, benefits from candidate $P$ 's platform independent who helped $P$ win, poor or rich voters. ${ }^{8}$

$I$ can be seen as a club good, the reward given to members of the social group that forms the electoral majority support for the victorious contender, representing the allocation of a scarce resource exclusively to that group. In the real world, such policies may allocate funds to an industry that is located where a critical mass of a supportive social group resides or protect an exclusive right valued by that social group. Mining subsidies for conservative whites in West Virginia are illustrative of the first type of policy, while things like exemption from military service for ultraorthodox Jews in Israel, policies that set official languages in multi-lingual societies, or immigration regulations that restrict resident permits to non-universal groups are examples of the second type. ${ }^{9}$

Even when $i$ does not vote for the winning candidate, she may receive the group benefit - if her group casts the most votes for the winning candidate. Suppose voter $i$ is member of $M J$ and the two other members of $M J$ as well as one member of MI vote for $P$ but voter $i$ herself votes for $R$. Then, $M J$ casts the most votes for the winning candidate, and all members of $M J$, including voter $i$, receive the group benefit.

I assume that the distribution $F\left(\omega_{i}\right)$ is contingent on identity group membership. There are three critical values in the income-space that will play an important role in the analysis. Let $\omega_{L} \equiv \frac{V-I}{\tau}, \omega_{M} \equiv \frac{V}{\tau}$, and $\omega_{H} \equiv \frac{V+I}{\tau}$ where $\omega_{L}<\omega_{M}<\omega_{H}$. I will refer to incomes below $\omega_{L}$ as very poor, those between $\omega_{L}$ and $\omega_{M}$ as moderately poor, those between $\omega_{M}$ and $\omega_{H}$ as moderately rich, and those above $\omega_{H}$ as very rich. Throughout the main analysis, I will assume that $\omega_{i}<\omega_{M}$ for all members of $M I$ and one member of $M J$ and that $\omega_{i}>\omega_{M}$ for the other two members of $M J$. In words, all members of $M I$ are either moderately or very poor while $M J$ is composed of one poor and two rich members. ${ }^{10}$

Finally, I assume when $i$ is indifferent between voting for candidate $P$ and candidate $R$, holding

\footnotetext{
${ }^{8}$ Income here is just one representation of an individual-level attribute that determines who benefits from the different candidates. It would certainly be conceivable that in some electoral contest, income is the basis of a grouptargeted benefit and cold be considered a group identity (i.e., class) as defined in this model.

${ }^{9}$ Politicians allocating resources to discernible social groups make each voter pivotal in the fight for such a targeted benefit. This also helps explain why turnout is often high, even though the influence of each individual vote on the overall electoral outcome is minuscule, and it illuminates why some voters appear to make choices that run counter to their obvious economic interests to secure a prize for their identity group (Morton, 1991; Schram and Sonnemans, 1996; Smith and Bueno De Mesquita, 2012).

${ }^{10}$ I present a more general version of this game with $F\left(\omega_{i}\right)$ orthogonal to identity group membership in Section A.2 in the online Appendix.
} 
fixed the rest of the strategy profile, $i$ votes for the candidate that would give $i$ a higher $U_{i}^{C}$.

Equilibrium Analysis I will define equilibrium strategy profiles of this game to be inclusive of mixing strategies and denote them by $\alpha^{*}=\left(\alpha_{1}^{* \mathrm{MJ}}, \alpha_{2}^{* \mathrm{MJ}}, \alpha_{3}^{* \mathrm{MJ}} ; \alpha_{1}^{* \mathrm{MI}}, \alpha_{2}^{* \mathrm{MI}}\right)$, where $\alpha_{i}^{* G} \in \Delta A_{i}$ with $G \in\{M I, M J\}$ assigns non-negative weights to the two elements of $A_{i}$ such that the weights sum to 1 for each $i$. In pure strategy equilibria, these weights are degenerate.

I focus on Nash equilibria in weakly undominated strategies. In particular, every equilibrium strategy $\alpha_{i}^{* G}$ of this game satisfies two conditions for every $i, G$ :

(1) There exists no $\alpha_{i}^{G} \in \Delta A_{i}, \alpha_{i}^{G} \neq \alpha_{i}^{* G}$ such that $u_{i}\left(\alpha_{i}^{G}, \boldsymbol{\alpha}_{-i}\right) \geq u_{i}\left(\alpha_{i}^{* G}, \boldsymbol{\alpha}_{-i}\right) \forall \boldsymbol{\alpha}_{-i} \in \boldsymbol{\Delta}_{\boldsymbol{A}_{i}}$ with strict inequality for some $\boldsymbol{\alpha}_{-i} \in \boldsymbol{\Delta} \boldsymbol{A}_{-i}$.

(2) There exists no $\hat{\alpha}_{i}{ }^{G} \neq \alpha_{i}^{* G}$ such that $u_{i}\left(\hat{\alpha}_{i}^{G}, \boldsymbol{\alpha}_{-i}^{*}\right)>u_{i}\left(\alpha_{i}^{* G}, \boldsymbol{\alpha}_{-i}^{*}\right)$.

Below I will refer to $\alpha^{*}$ that meet these conditions as "equilibria."11

Proposition 1 The only pure strategy equilibria possible are of the form $(P, P, P ; P, P)$ and $(R, R, R ; P, P)$. The $(P, P, P ; P, P)$ equilibrium always exists. The $(R, R, R ; P, P)$ equilibrium and an equilibrium in mixed strategies of the form $\left(\alpha_{1}^{* M J}(P), \alpha_{2}^{* M J}(P), \alpha_{3}^{* M J}(P) ; P, P\right)$, where $\left(\alpha_{1}^{* M J}(P), \alpha_{2}^{* M J}(P), \alpha_{3}^{* M J}(P)\right)$ are probabilities of playing $P$ for players $i=\{1,2,3\} \in M J$ respectively, exist if and only if all members of MJ are not very poor, i.e. if their incomes are higher than $\omega_{L}=\frac{V-I}{\tau} .{ }^{12}$

Given that this is a coordination game, it should not be surprising that there are multiple Nash equilibria in pure strategies satisfying equilibrium conditions (1) and (2). For convenience, I will refer to the $(P, P, P ; P, P)$ equilibrium (where $P$ wins the election) as $P$-equilibrium and the $(R, R, R ; P, P)$ equilibrium (where $R$ wins the election) as $R$-equilibrium. Proposition 1 provides a clear prediction if at least one member of $M J$ is very poor: in the $P$-equilibrium, members of $M J$ solve the trade-off between the individual-level and group-targeted benefit by all voting for the redistributive candidate $P$ even though this imposes a large cost on the rich members of $M J$ not made up for by securing the group-targeted benefit $I$. If no member of $M J$ is very poor, Proposition 1's equilibrium prediction for members of $M I$ is also unambiguous, vote for the redistributive candidate $P$, but for $M J$ multiple equilibrium sub-profiles exist. Still, there is a group-welfare

\footnotetext{
${ }^{11} \mathrm{~A}$ proof for the proposition in this section is provided in Section A.1 of the online Appendix.

${ }^{12}$ The values for $\alpha_{i}^{* M J}$ are given in the appendix.
} 
ordering of these equilibrium sub-profiles: $M J$ 's welfare is maximized when all members vote for the wealth-preserving candidate $R$. Note, both coordination mechanism, equilibrium coordination on candidate $P$ and group-majoritarian coordination where $M J$ votes for $R$ and $M I$ for $P$, correspond to equilibria of the simple model of electoral competition but the latter only exists if no member of $M J$ is very poor.

\section{Experimental design}

The experiment varies the distribution of income within groups (group heterogeneity treatments) to tests whether behavior congruent with equilibrium predictions arises. It also introduces primes of either the individual-level or group-targeted benefit component of voters' utility function (appeal treatments) to assess the robustness of equilibrium play and to identify voters' decision-making mechanism, group-majoritarian or equilibrium coordination.

In the experiment, I simulate vote choice between two candidates and if chosen by electoral majority, one candidate implements redistribution, while the other implements a status-quo distribution. Following the model presented in Section 3, I will refer to the former as "candidate P" and the latter as "candidate R." On the subject screens in the experiment, candidate $P$ is referred to neutrally as Alternative $A$ and candidate $R$ as Alternative $B$ as to not add a priming effect. Additionally, the winning candidate rewards the group that casts the most votes for her among all voters who support her by implementing a policy that will disproportionally allocate a scarce resource to that group. Voters are characterized by two social attributes: individual "income" and membership in a "social group." While income outside of the laboratory could constitute membership in a social group (e.g. the poor, middle class, etc.), social group membership here is defined by the attribute that determines receipt of the group-targeted benefit. I will refer to membership in that social group as group identity, and to the other as individual-level attribute (assigned income).

Each experimental session unfolds in two stages: (1) group identity inducement stage and (2) voting game stage. The voting game stage runs for 40 rounds. ${ }^{13}$

\footnotetext{
${ }^{13}$ The experiment was not pre-registered. All treatments conducted within the research agenda are presented in the main text or the online Appendix and no observations are omitted.
} 
Group identity inducement stage At the beginning of each experimental session, subjects are assigned to be a Klee or a Kandinsky following standard procedure for inducing group identities in experiments. ${ }^{14}$ This assignment constitutes their membership in a social group and a groups' status as majority $M J$ or minority $M I$ is randomly assigned to the social groups of Klees and Kandinskys.

In the subsequent voting game stage, the identities of all subjects with whom individual subjects interact are displayed for them on the screen, making the artificially induced group identities salient. Group membership is directly payoff relevant adding to the salience of group identities in the experiment ${ }^{15}$ and implying a potential experimenter demand effect. I experimentally account for some consequences of such demand effects in the analysis (See Section 5.4) but note that other demand effects are a desired feature of the experimental design. ${ }^{16}$

Voting game stage The voting game proceeds as follows: ${ }^{17}$

1. Subjects are randomly assigned to a 5-person society at the beginning of the session and that assignment stays fixed until the end of the experiment.

2. In each round, subjects are randomly assigned their income from the underlying set of fixed income distributions without replacement. ${ }^{18}$

3. Subjects are informed about income and group identities of all subjects in their society.

4. Subjects are asked to make a choice between two candidates, $P$ and $R$. Whichever candidate receives the most votes in their society becomes the winning candidate of that society.

5. The winning candidate is announced to the members of the society and subjects are privately informed about their round payoffs.

In this experiment, $\tau=1 / 2, V=25$, and $\mathcal{I}=10$, which ensures existence of equilibria described in Section 3 and allows for easily comprehendible cut-points in the income space. The round payoff to subject $i$ when $P$ wins is given by

\footnotetext{
${ }^{14}$ See Section B in the online Appendix for details on the procedure which follows Tajfel and Billig (1974), Chen and Li (2009), and Landa and Duell (2015). Considerable experimental literature using the minimal group paradigm has shown its effectiveness in inducing patterns of responses to identity, including in-group favoritism and inter-group competition, that resemble those observed outside the laboratory with naturally occurring groups (e.g., Eckel and Grossman (2005)). The minimal group design does not attempt to manipulate something "seemingly immutable" (Sen and Wasow, 2016) but induces minimal groups avoiding uncontrolled associations (Tajfel and Turner, 1986).

${ }^{15}$ While explicitly announcing group membership is in line with a large literature in experimental social science employing the minimal group design, making the minimal group assignment payoff relevant is less so but still often implemented (e.g., Fryer, Goeree and Holt (2005)).

${ }^{16}$ The group appeal models an electoral campaign appeal that asks voters explicitly to consider preferences and behaviors of fellow group members more strongly.

${ }^{17}$ Section B.4 in the online Appendix shows instructions and subject screens of the experiment.

${ }^{18}$ Section B.5 in the online Appendix gives the full set of income distributions.
} 
$\operatorname{payoff}_{i}(\mathrm{P}$ wins $)=\left\{\begin{array}{l}\frac{1}{2} \text { income }_{i}+25+10 \\ \frac{1}{2} \text { income }_{i}+25\end{array}\right.$ if $i$ 's group holds a majority among all voters who vote for candidate $P$ otherwise

The round payoff to subject $i$ when $R$ wins is given by

$$
\text { payoff }_{i}(\mathrm{R} \text { wins })= \begin{cases}\text { income }_{i}+10 & \text { if } i \text { 's group holds a majority } \\ & \text { among all voters who vote for candidate } R \\ \text { income }_{i} & \text { otherwise }\end{cases}
$$

It is made clear to subjects that there are two distinct parts to the their round payoff: the individual-level benefit that depends on their assigned income and the group-targeted benefit that is determined by whether $i$ 's group holds a majority among the supporters of the winning candidate.

The list of feasible income values potentially assigned to each subject contains $10,22,27,38$, $44,56,62,73$, and 90 . In what follows (but not on the subjects' screen within the experiment), voters are defined as "very poor" if they are assigned the three lowest possible values of income (10, 22 , or 27 ), "moderately poor" if they have the next two income values (38 or 44), "moderately rich" if they are assigned the two following values (56 or 62), and "very rich" if they have one of the two highest income values (73 or 90). Three out of five voters in a society are either very or moderately poor with an assigned income below 50, but two out of three members of $M J$ are moderately or very rich with an assigned income above 50 .

The payoffs in the game are structured so that the loss in individual-level benefit for a moderately poor (rich) voter when $R(P)$ wins is more than offset when her group secures the grouptargeted benefit but the very rich and the very poor voters would prefer receiving the individual-level to the group-targeted benefit if only one is to be had.

Treatments Group heterogeneity treatments vary the distribution of assigned income within groups (within subject-design). Appeal treatments vary whether the individual-level or grouptargeted benefit component in subjects' utility function is visually primed on the subjects' screen. Appeal treatments are assigned on the sessions-level (between subject-design).

I implement three main group heterogeneity treatments that vary the level of income heterogeneity in the larger social group $M J$. In the low group heterogeneity treatment, $M J$ is composed of one very rich, one moderately rich, and one moderately poor voter while in the smaller social 
group $M I$ there is always one moderately poor and one very poor voter. In the medium group heterogeneity treatment, the poorest voter in $M J$ is very poor and in the high group heterogeneity treatment, that poorest voter's income is much lower and income of the richest voter in $M J$ is much higher than in the medium or low group heterogeneity treatments. Variation induced by these treatments is a feature of $M J$; the distribution of incomes in $M I$ is constant. Figure 1 summarises the within-society distribution of income across group heterogeneity treatment conditions. ${ }^{19}$

Figure 1: Distribution of income in majority $M J$ and minority $M I$ (gray markers) within societies by group heterogeneity.

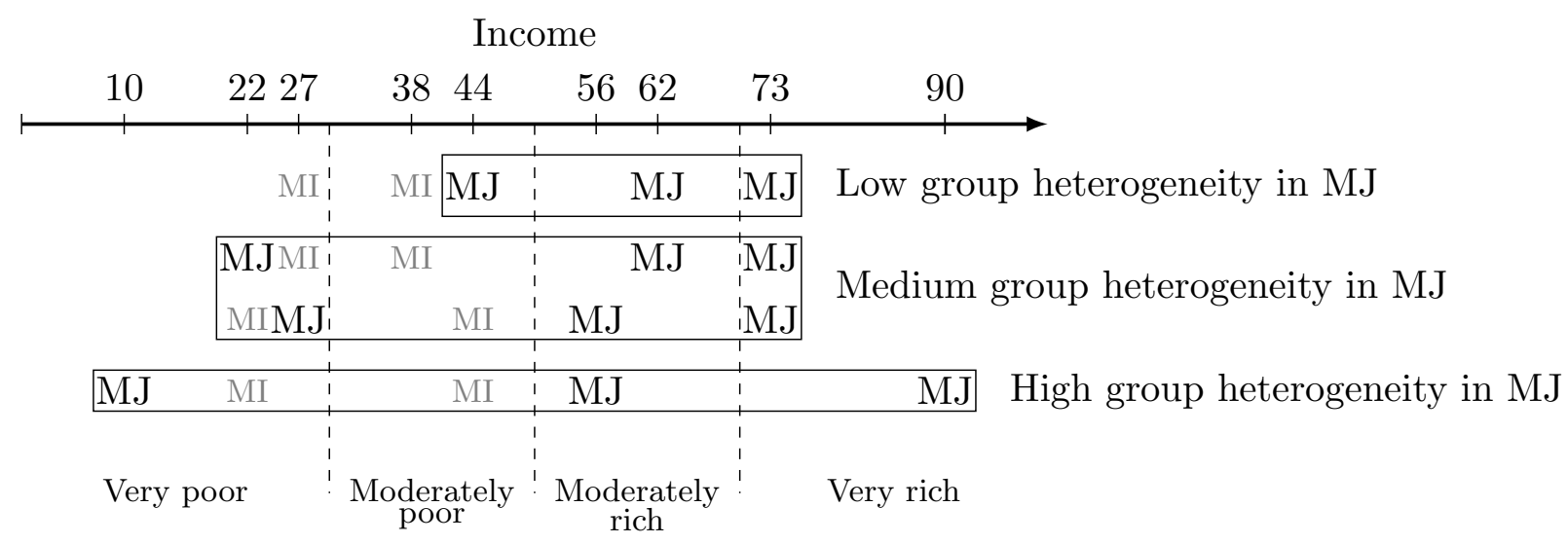

In the no appeal treatment there is no priming, in the group appeal treatment the necessity to coordinate with fellow group members to secure the group-targeted benefit is highlighted, and in the income appeal treatment voters' individual-level attribute income is primed. Appeals are shown to subjects on their computer screens while they are making their voting decisions. The statement representing a group appeal, depending on Klee [Kandinsky] group membership, reads: Remember you are a KLEE [KANDINSKY]! Should you vote with other Klees [Kandinskys], you may receive a higher identity payoff. The income appeal reads: Remember your income is below 50! Should you vote for Alternative A, you may receive a higher decision payoff. ${ }^{20}$ The income appeal treatment provides a clear behavioral prescription contingent on income while the group appeal treatment only reminds subjects that the groups' coordination would be necessary to secure the

\footnotetext{
${ }^{19}$ Small perturbations of income in the medium group heterogeneity treatment are introduced to keep subjects alert.

${ }^{20}$ This appeal is shown when the subject is assigned an income below 50 . When the subject is assigned an income above 50, the statement reads: Remember your income is above 50! Should you vote for Alternative B, you may receive a higher decision payoff. The individual-level benefit was referred to as "decision payoff" in the experiment.
} 
group-targeted benefit but the treatment does not name the target of such coordination.

For robustness checks and for additional tests to identity treatment effects, I implement a series of supplemental treatments that are described in the results section once referenced. ${ }^{21}$

Table 1: Number of societies, subjects, and subject-round observations for appeal and group heterogeneity treatment conditions. Every session includes 8 rounds of the low group heterogeneity, 28 of the medium group heterogeneity, and 4 rounds of the high group heterogeneity treatment.

\begin{tabular}{|c|c|c|c|c|c|c|}
\hline \multirow[t]{3}{*}{ Appeal treatments } & \multirow[t]{3}{*}{ Societies } & \multirow[t]{3}{*}{ Subjects } & \multicolumn{4}{|c|}{ Subject-round observations } \\
\hline & & & & by level c & group hete & ogeneity \\
\hline & & & $\begin{array}{c}\text { Total } \\
\text { (40 rounds) }\end{array}$ & $\begin{array}{c}\text { Low } \\
\text { (8 rounds) }\end{array}$ & $\begin{array}{c}\text { Medium } \\
\text { (28 rounds) }\end{array}$ & $\begin{array}{c}\text { High } \\
\text { (4 rounds) }\end{array}$ \\
\hline No appeal & 14 & 70 & 2800 & 560 & 1960 & 280 \\
\hline Group appeal & 16 & 80 & 3200 & 640 & 2240 & 320 \\
\hline Income appeal & 8 & 40 & 1600 & 320 & 1120 & 160 \\
\hline Total & 38 & 190 & 7600 & 1520 & 5320 & 760 \\
\hline
\end{tabular}

The appeal treatments are subtle but are perceived by subjects to influence behavior ${ }^{22}$ and treatment conditions are balanced in observables. The number of independent observations in the study is equal to the number of societies of 5 voters and accounted for in the statistical analysis accordingly. While the order of rounds in which a particular level of group heterogeneity (low or medium) occurred was randomly drawn, the order remained the same during all sessions with high group heterogeneity being implemented in the last four rounds. This design was chosen to maximize the power of statistical tests of the effects of the appeal treatments, which were expected to be much smaller than the effects of the group heterogeneity treatments. In this way, order effects may arise.

Hypotheses Testing for the prevalence of different equilibria, I facilitate variation in the distribution of income assigned to members of $M J$ as induced by the group heterogeneity treatments. Equilibrium predictions derived in Section 3 lead directly to my first hypothesis:

Hypothesis 1 (Equilibrium predictions) When group heterogeneity of the majority $M J$ of a society

\footnotetext{
${ }^{21}$ More detailed descriptions, summary statistics of the main variables, treatment statistics for the full set of treatments, and balance statistics are shown in Section B of the online Appendix.

${ }^{22}$ Eighty-nine percent of subjects in group or income appeal conditions responded to the exit-survey question whether appeals affected their decisions with an answer that is not "I have not recognize any appeal." Further, 79\% responded they have seen the appeal when asked directly (data collected in 3 out of 12 sessions).
} 
is medium or high, the minority $M I$ and the majority $M J$ will always support the redistributive candidate $P$ and $P$ wins the election (P-equilibrium). When group heterogeneity of the majority $M J$ is low, the majority $M J$ also votes for the wealth-preserving candidate $R$ while the minority MI still chooses $P$ (R-equilibrium).

In both cases, $M J$ receives the group-targeted benefit, but in the latter the $P_{-}, R$-, and mixedstrategy equilibrium exists as stated by Proposition 1. Given the parameters implemented in the experiment, in the a mixed-strategy equilibrium the moderately poor member of $M J$ chooses candidate $R$ with a probability of .38 , the moderately rich member with a probability of .62 , and the very rich member with a probability of .74, and $M I$ chooses candidate $P$ for sure.

I empirically assess hypothesis 1 at the society-level where the equilibrium strategy profile is defined and compute the relative frequency of the possible strategy profiles and compare their occurrences across group heterogeneity treatments. ${ }^{23}$ Observations on the presented statistics across levels of group heterogeneity come from repeated measurements taken within each society. ${ }^{24}$

While variation in group heterogeneity leads to clear hypotheses with respect to which equilibrium should be prevalent, identifying how robust equilibria are to the ways voters trade off individual-level and group-targeted benefit components of their utility function is less straightforward. A unique equilibrium exists when group heterogeneity in $M J$ is medium, but playing that $P$-equilibrium and securing the group-targeted benefit imposes hefty costs on the rich members of $M J$. The same equilibrium prediction applies for the high heterogeneity treatment, but the potential loss in individual-level benefit when voting for $P$ as the richest member of $M J$ increases even more. Are those rich voters always willing to accept a loss in the individual-level dimension to win the club good as predicted in the equilibrium? Here is where the appeal treatments allow identification of how much equilibrium play is affected by voters assigning higher weight to either

\footnotetext{
${ }^{23}$ Whenever I present tests over statistics computed at the society-level (i.e., relative frequency of strategy profiles) and claim significance, (1) Wilcoxon sign rank-tests lead to rejection of the null hypothesis at $\alpha=.05$ (if not other pvalue is provided); and (2) the $95 \%$ confidence bounds of a society-level clustered bootstrap of the difference in relative frequency do not contain zero. I print one-sided tests when the hypothesis tested makes a directional prediction. I provide further analysis in a regression framework allowing for a richer model of subject-, group-, and society-level effects in Section C.2 of the online Appendix).

${ }^{24}$ Note that the voting game in the experiment constitutes a repeated interaction while the model in Section 3 is a one-shot game. When group heterogeneity in $M J$ is low, interpreting the observed relative frequency of strategy profiles across all rounds as indicating the prevalence of a particular equilibrium of the one-shot game, might be reflecting strategy profiles consistent with an equilibrium in a finitely repeated game instead. When group heterogeneity in $M J$ medium or high, this concern is mute, because a finitely repeated game would deliver the same unique equilibrium prediction.
} 
the individual-level or group-targeted benefit component of their utility function. Recall that the income appeal gives a specific behavioral prescription based on subjects' level of assigned income: vote for candidate $R$ if you are rich and vote for candidate $P$ if you are poor. The group appeal, in contrast, reminds subjects of the necessity to coordinate with fellow group members to secure the group-targeted benefit; this appeal does not promote a specific target for coordination.

With an income appeal, should the appeal be effective, the $R$-equilibrium is less likely to occur because the poor member of $M J$ is more prone to deviate to $P$. The $P$-equilibrium however, will not arise more often because the rich members of $M J$ are at least as likely, if not even more likely, to vote for candidate $R$. Further, an increased propensity to vote with fellow group members upon receiving a group appeal is conceivable as long as the target of coordination is not ambiguous. Regarding the prevalence of equilibrium play, this means that because the $P$-equilibrium is the unique equilibrium, it is more likely to be played in the group appeal than in the no appeal treatment. When group heterogeneity of $M J$ is low, multiple equilibria exist with different behavioral prescriptions for members of $M J$, and no clear effect of priming the benefits of group coordination arises.

The hypothesis below aggregates the predictions about the effects of appeal treatments:

Hypothesis 2 (Appeal treatment effects) Priming the individual-level benefit splits voters by income therefore decreasing the prevalence of any equilibrium. Appealing to consider the group-targeted benefit makes all voters more prone to vote for redistributive candidate $P$ when group heterogeneity in the majority is not low and increases the likelihood that the P-equilibrium is played.

To evaluate hypothesis 2, I test for changes in the relative frequency of equilibrium profiles played and changes in the vote share of candidate $R$ across appeal treatments. Additionally, looking at vote share captures marginal changes in support for candidates - even those that do not imply that societies switch to or away from an equilibrium. ${ }^{25}$ When testing for group appeal treatment effects, observations on the presented statistics come from measurements taken in independent samples of societies assigned to the different treatment conditions.

Hypothesis 2 arises from variations in voter behavior that are rooted in voters weighing either the individual-level or group-targeted benefit component of their utility function higher upon re-

\footnotetext{
${ }^{25}$ I follow a similar procedure as with the empirical tests of hypothesis 1, substituting sign rank- with Wilcoxon rank sum-tests and subject-level clustered bootstrap where appropriate. Note, evaluating the expected effects of the appeal treatments is not a comparative static-type of analysis arising from the model; it is an analysis of the effects of a priming treatment on behavior.
} 
ceiving an appeal. While the effects of priming income seem straightforward in guiding vote choice, when asked to consider coordinating with fellow group members, voters should immediately wonder what is the target of coordination to maximize the probability of receiving the club good. To answer that question satisfactorily, voters must, upon receiving a group appeal, either formulate expectations about fellow group members' and the other groups' behavior or they must follow a simple decision-heuristic.

In both pure strategy equilibria, $M J$ wins the club good, but the $P$-equilibrium proves more costly to the rich majority of members of $M J$. This implies a group welfare ordering of equilibria favoring coordination on the $R$-equilibrium. ${ }^{26}$ Such coordination on the $R$-equilibrium that maximizes $M J$ 's group welfare represents behavior that follows the logic of group-majoritarian coordination, as introduced earlier. Following the heuristic of group-majoritarian coordination however, can arise in non-equilibrium play in the form of the strategy profile $(R, R, R ; P, P)$ when group heterogeneity in $M J$ is medium or high; it only requires voters to choose what is best for the group defined as voting for the candidate whose platform is beneficial to most members of the group.

Whether equilibrium coordination happens with group appeals is identified when voters coordinate on candidate $P$ and when their vote for $P$ is caused by voters forming the expectation that fellow group members and the members of the other group will vote for $P$ as well.

Hypothesis 3 (Coordination mechanism) Group appeals trigger equilibrium coordination if voters coordinate on voting for the redistributive candidate $P$ and make their decisions in response to the expectations that other voters will also vote for P. They trigger group-majoritarian coordination if voters in the majority $M J$ coordinate on voting for the wealth-preserving candidate $R$ independent of their income and the level of group heterogeneity.

Observing equilibrium coordination is equivalent to observing the $P$-equilibrium, while observing group-majoritarian coordination requires the strategy profile $(R, R, R ; P, P)$ to be played (whether in equilibrium or not). Identifying group-majoritarian coordination empirically is straight-

\footnotetext{
${ }^{26}$ To illustrate, given the parameters of the game implemented in the laboratory, when group heterogeneity in $M J$ is low and its members manage to secure the full group-targeted benefit from candidate $R$, the sum of the individual's payoffs of all members of $M J$ is maximized. Coordinating on $P$ instead gives them about $\$ 2$ less. When group heterogeneity in $M J$ is medium, switching the coordination target in this way still reduces the group's sum of payoffs but only by about 50 cents. A deviation of one of the members of $M J$ away from either of the equilibria in pure strategies, which would have secured the group-targeted payoff for $M J$, cost the group about $\$ 2.5$ and $\$ 5$ when two members of $M J$ deviate. Table B.4 in the online Appendix provides subject payoffs in tokens that represent the individual-level benefit from each of the candidates.
} 
forward - a positive treatment effect of group appeals (over no appeals) on the likelihood that $M J$ votes for candidate $R$ independent of assigned income and level of group heterogeneity. The empirical test for the existence of equilibrium coordination, in contrast, is composed of two steps: First, I must identify $M J$ groups likely to vote for $P$ and, second, evaluate whether the decisions of members of such $M J$ groups are based on forming beliefs about others' choices.

\section{Results}

In the analysis of experimental data to follow, I first characterize the relative frequency of equilibrium play as a function of the group heterogeneity treatments. Second, I provide average treatment effects of the appeal treatments on the relative frequency of equilibrium play as well as on the average vote share of candidate $R$. Finally, I provide evidence for the mechanism behind the effects of group appeals (identifying equilibrium and group-majoritarian coordination) and discuss the robustness of experimental results.

\subsection{Equilibrium predictions}

When group heterogeneity of $M J$ is low, societies choose the $R$-equilibrium in $45 \%$ of elections. In this equilibrium all members of $M J$ vote for the redistributive candidate $R$, all members of $M I$ vote for wealth-preserving candidate $P, R$ wins the election, and $M J$ earns the group-targeted benefit $I$. The distribution of strategy profiles as well as the distribution of which candidate won the election is shown in Figure 2; observations for when group heterogeneity in $M J$ is low appear in the left panel. The relative frequency of the $P$-equilibrium where all voters choose $P$ is shown on top (blue, darkest color); the strategy profile where $M J$ votes for candidate $R$ and $M I$ supports $P$ is given at the bottom (red, second darkest color). The relative frequency of profiles in which $P$ wins but are not the $P$-equilibrium, is given in the lightest color (light blue); the profile where $R$ wins, except $(R, R, R ; P, P)$, is shown in the second lightest color (light red). At this level of group heterogeneity, averaging across appeal treatments, only rarely do societies converge to the $P$-equilibrium (relative frequency .02), candidate $R$ wins in $67 \%$ of elections, and $M J$ earns the group-targeted benefit in $72 \%$ of the elections (and shares it with $M I$ in $12 \%$ ).

When group heterogeneity in $M J$ is medium or high, the $R$-equilibrium does not exist and, 
Figure 2: Distribution of relative frequency of strategy profiles by group heterogeneity and appeal treatments.
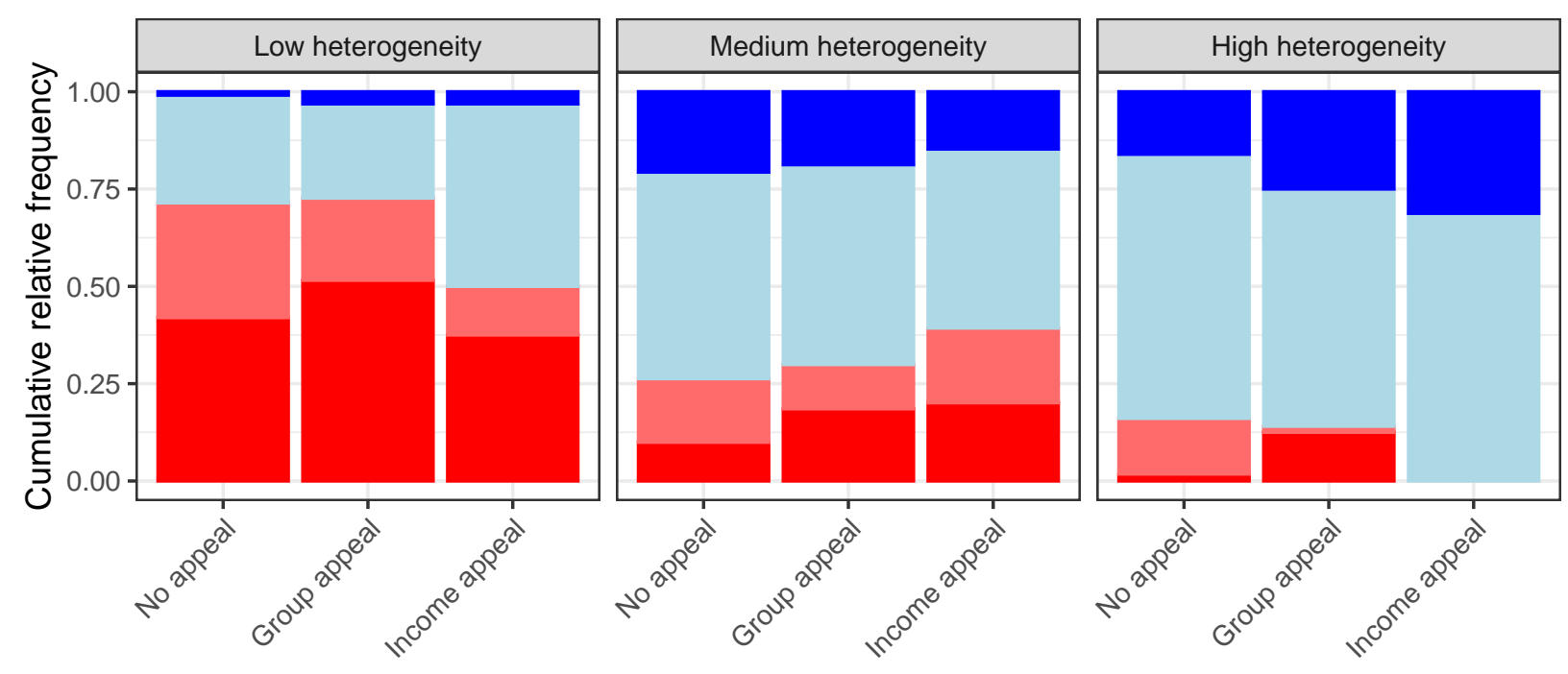

Redistributive cand. $\mathrm{P}$ wins, all vote $\mathrm{P}$ Redistributive cand. $\mathrm{P}$ wins, MJ or MI split

unsurprisingly, the strategy profile where all members of $M J$ coordinate on candidate $R$ and all members of $M I$ vote for candidate $P$ occurs significantly less often (at a rate of .16 and .06, respectively). The relative frequency of the $P$-equilibrium, however, increases significantly; from .02 when group heterogeneity in $M J$ is low, to .19 when it is medium, and to .23 when it is high. When group heterogeneity in $M J$ is medium or high, candidate $R$ wins the majority in only $31 \%$ and $12 \%$ of elections, which is a significant drop of $37 \%$ and $56 \%$ from when group heterogeneity in $M J$ is low, respectively. $M J$ receives the full group-targeted benefit only in $57 \%$ and $45 \%$ of elections, a decline of $15 \%$ and $27 \%$, respectively.

I do not find evidence that societies, which do not play either of the two pure strategy equilibria when group heterogeneity is low, choose congruent with the mixed strategy equilibrium; in particular the moderately poor and the moderately rich member of $M J$ in such societies vote for candidate $R$ significantly too often (at a rate of .67 and .75 , in contrast to the predicted .38 and .62 , respectively).

Result 1 When group heterogeneity is low, the majority MJ is more likely to coordinate on the wealth-preserving candidate $R$, helping $R$ win, and taking the group-targeted benefit. When group heterogeneity is medium or high, the majority MJ tends to fully coordinate on candidate $R$ or the 
redistributive candidate $P$ at similar rates, but given a higher frequency of split votes and because the minority $M I$ always votes for $P$, candidate $P$ wins most elections.

\subsection{Appeal treatment effects}

When group heterogeneity is low, the relative frequency of the $R$-equilibrium does not vary significantly across appeal treatments: it is .42 in the no appeal treatment, .52 in the group appeal treatment, and .38 in the income appeal treatment. Testing for differences in no appeal vs group appeal (income appeal) treatments returns $p=.15(p=.69)$. Findings of no treatment effect of the group and income appeal also emerge for the frequency of the $P$-equilibrium and frequency with which either candidate wins the election for all levels of group heterogeneity. In societies where group heterogeneity in $M J$ is medium or high, the non-equilibrium strategy profile $(R, R, R ; P, P)$ is played more often in income $(p=.11)$ and significantly more often in group appeal than no appeal treatment (difference .09 each).

In the income appeal treatment, the vote share of candidate $R$ among the poor member of $M J$ is by $.11, .24$, and .28 lower than among rich members when group heterogeneity is low, medium, and high, respectively (where the difference between poor and rich is significant for the latter two levels of group heterogeneity). The difference between poor and rich in $M J$, however, is stable across all appeal treatments; see Figure 3, which shows the vote share of candidate $R$ across group heterogeneity and appeal treatments for rich $M J$ (black bars), poor $M J$ (white bars), and $M I$ (gray bars).

The vote share of candidate $R$ in $M I$ is low throughout but falls significantly in the group appeal over the no appeal treatment when group heterogeneity is medium or high while the income appeal has no such effect.

The following summarises appeal treatment effects on equilibrium play and vote choice. ${ }^{27}$

Result 2 Income and group appeal treatments do not affect the frequency of equilibrium play. When group heterogeneity in the majority $M J$ is medium or high, both appeals increase the likelihood that

\footnotetext{
${ }^{27}$ There is also no average appeal treatment effect on efficiency when group heterogeneity in $M J$ is low because no effect of group appeals arises. When group heterogeneity in $M J$ is medium or high, group appeals make $M I$ significantly more likely to vote for candidate $P$, but also some of the $M J$ groups more likely to fully coordinate on $P$; in this way, the average payoffs from securing the group-targeted benefit from $P$ as well as its distribution across $M I$ and $M J$ remains the same, canceling out any appeal treatment effect on welfare.
} 
Figure 3: Vote share of the wealth-preserving candidate $R$ by group heterogeneity and appeal treatments for majority $M J$ and minority $M I$. Confidence bars are computed from a subject-level clustered bootstrap.
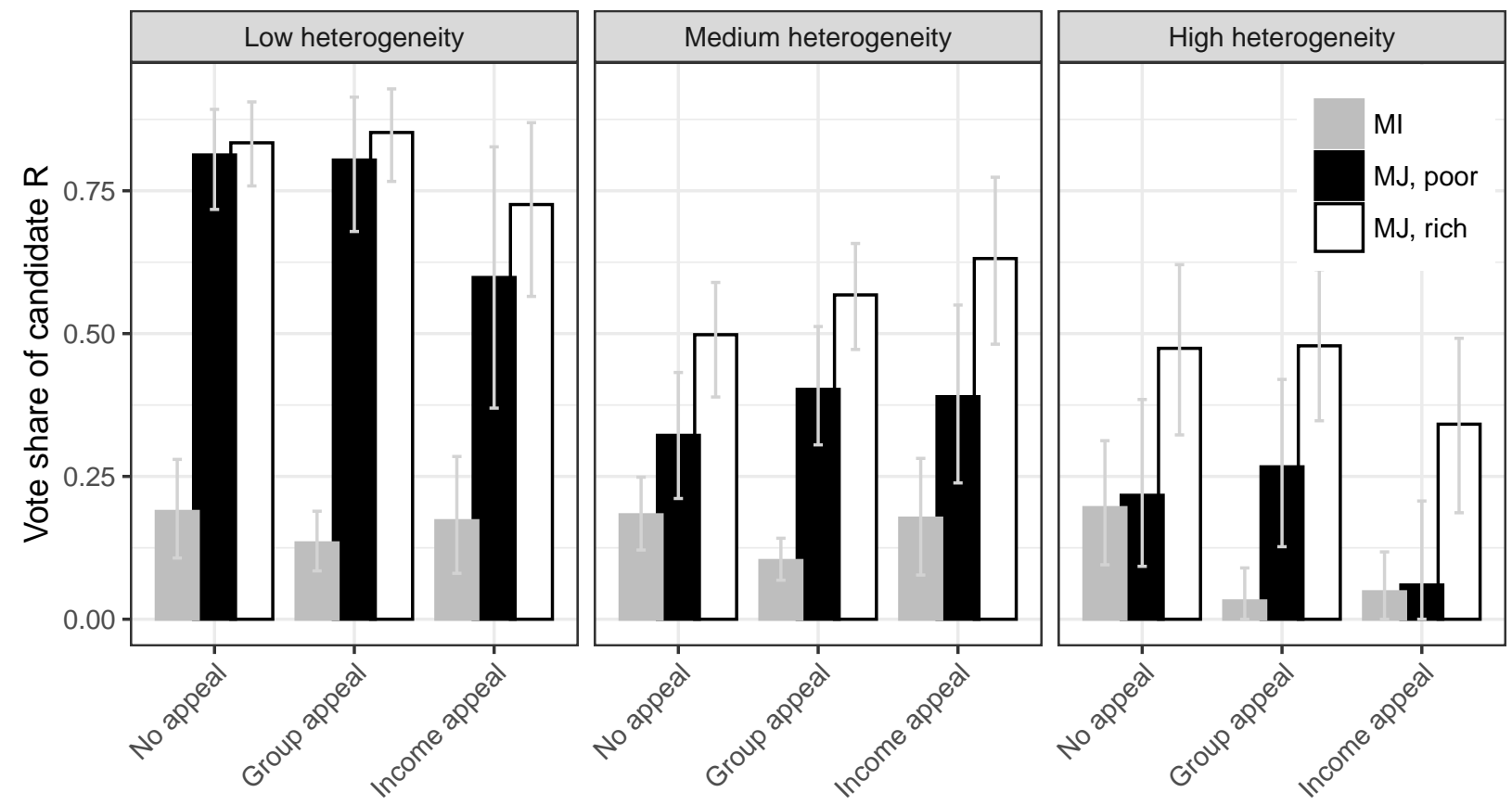

the wealth-preserving candidate $R$ wins with the support of the majority $M J$ while the minority $M I$ still votes for the redistributive candidate $P$ and $M I$ 's support for $P$ is strongest upon receiving a group appeal.

\subsection{Coordination mechanism}

Two interesting observations emerge from the analysis so far: (1) high frequency of the $R$-equilibrium when group heterogeneity in $M J$ is low and the $P$-equilibrium also exists, and (2) strong support for candidate $R$ when group heterogeneity $M J$ is medium or high and only the $P$-equilibrium $(P, P, P ; P, P)$ exists (in particular in income and group appeal treatments). In a decision situation with multiple equilibria (i.e., when group heterogeneity in $M J$ is low), behavior congruent with the simple heuristic provided by group-majoritarian coordination arises unsurprisingly; but why do we observe behavior equivalent to using such heuristic when the behavior is not congruent with equilibrium play? And, which of the societies playing the $P$-equilibrium truly follow equilibrium coordination? 
To identify group-majoritarian and equilibrium coordination, I look at instances of medium or high group heterogeneity in $M J$ and I define $M J$ with a propensity to coordinate on $R$ by when $M J$ shows an average rate of voting for candidate $R$ that is higher than the average vote share of $R$ of all $M J$ in a given treatment group. And, I define $M J$ with a propensity to coordinate on $P$ by when $M J$ displays an average vote share of candidate $R$ that is lower than the average vote share of $R$ of all $M J$ in a given treatment. I find sizable variation in whether $M J$ in the different societies coordinate on the candidates even within treatment condition. In the no appeal treatment, $43 \%$ of $M J$ groups show a propensity to coordinate on $R$ while that percentage grows to $50 \%$ in the income appeal treatment and $56 \%$ in the group appeal treatment; the remainder of $M J$ groups in each treatment condition displays a propensity to coordinate on $P$.

The vote share of candidate $R$ in $M J$ groups showing a propensity to coordinate on $R$ in the no appeal treatment is by .46 significantly higher than the vote share of $M J$ groups with a propensity to coordinate on $P$; the difference is .50 in the group and income appeal treatments. $M J$ with a propensity to coordinate on $R$ may follow the logic behind group-majoritarian coordination. $M J$ with a propensity to coordinate on $P$ may engage in equilibrium coordination.

Figure 4 illustrates the effect of group and income appeal on $M J$ 's coordination effort. Receiving the income appeal hinders coordination and $M J$ 's attempt to converge on candidate $R$ falls apart towards the end of the experiment (right, bottom panel). Making the poorest member of $M J$ recognize the cost of converging with fellow group members on $R$ with an income appeal clearly leads to coordination failure. The dynamics of vote share in $M J$ with a propensity to coordinate on $R$ in no appeal and group appeal treatment is quite similar (left and middle, bottom panel), which could be an indicator for how powerful (or simple) the heuristic behind group-majoritarian coordination is given that $M J$ does not even need the group appeal to trigger voting for candidate $R$.

Members of $M J$ are identified to choose according to group-majoritarian coordination, however, only if their support for candidate $R$, upon receiving a group appeal, is independent of their assigned income and the level of group heterogeneity. While the propensity to coordinate on candidate $R$ certainly varies with assigned income, the group appeal treatment effect on vote choice remains the same in size and direction and is significantly different from zero at all levels of income $(p<.1$ for comparisons at income 10,22, and 90). The true test of the existence of the 
group-majoritarian coordination heuristic, however, is to evaluate whether the poorest member of $M J$ supports $R$ at all levels of group heterogeneity. When group heterogeneity in $M J$ is medium or high, the loss in individual-level benefit for the poorest member is not offset by the group-level benefit received from the wealth-preserving candidate $R$; this is the only case where a vote for $R$ from the poorest member of the majority identity group would be not congruent with equilibrium play but still driven by the group welfare heuristics behind group-majoritarian coordination. With low group heterogeneity in $M J$, the (moderately) poor member support candidate $R$ at a rate of .81. When group heterogeneity in $M J$ is medium or high, however, the vote share of the wealth-preserving candidate among the poorest member of $M J$ drops significantly to .40 and .27, respectively.

Figure 4: Vote share of the wealth-preserving candidate $R$ for poor and rich members of the majority $M J$ by appeal treatment and coordination propensity (Medium and high group heterogeneity). Confidence bars are computed from a subject-level clustered bootstrap.
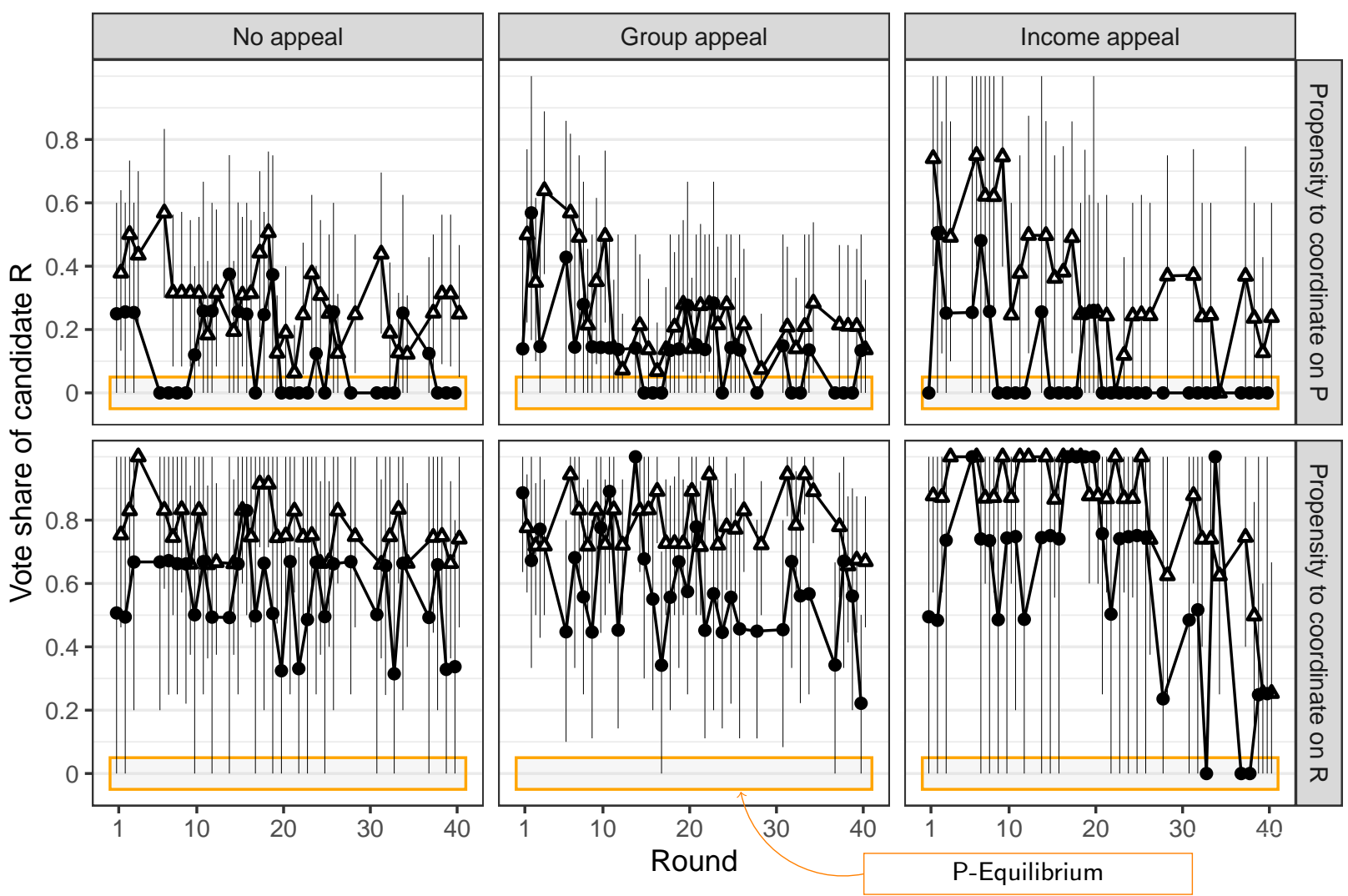

- Poor in MJ Rich in MJ

$M J$ members with a propensity to coordinate on $P$ may follow equilibrium coordination when 
coordination on candidate $P$ arises because they form expectations about the behavior of other voters as a result of the group appeal and choose accordingly. Several pieces of evidence support the finding that $M J$ groups with a propensity to coordinate on $P$ actually follow the logic of equilibrium coordination.

First, the P-equilibrium proves powerful in not only keeping the rich members of $M J$ to deviate to higher levels of support for candidate $R$ but to quickly converge to voting for $P$ as well. The top-middle panel of Figure 4 shows a significant time trend in average vote share of candidate $R$ for $M J$ with a propensity to coordinate on $P$ in the group appeal treatment, for rich and poor alike, while the average vote share of candidate $R$ remains stable for $M J$ with a propensity to coordinate on $R .^{28}$

Second, priming subjects' considerations of their individual-level attribute as implemented in the income appeal treatment, should directly induce rich members of $M J$ to expect that the poorest member is likely to side with the poor $M I$ in voting for candidate $P$. In turn, rich voters in $M J$ responding to that expectation vote for candidate $P$ given that $P$ is now more likely to be the vote winner. Indeed, when income is primed rich voters in $M J$ coordinate on candidate $P$ as the experiment proceeds. Clearly, in the income appeal treatment, quick coordination with fellow group members on $P$ is harder to achieve but happens eventually; the right panel in Figure 4 shows that rich members of $M J$ with a propensity to coordinate on $P$ hold off longer with their support for the redistributive candidate than that is the case in the group appeal treatment.

Third, the Rich $M I$-Group appeal treatment removes any belief that $M I$ is voting for candidate $P$ by creating a scenario in which all subjects in $M I$ are wealthy. The wealth-preserving candidate now receives support at a rate of .92 because the poorest member of $M J$ no longer sees a reason to expect $M I$ to vote for candidate $P$, and, in turn, the richer members of $M J$ are more certain that the poorest member coordinates with them on candidate $R$.

Finally, given that equilibrium coordination on the redistribution candidate $P$ relies on the beliefs of members of $M J$ about their poorest member and the expectation of the poorest member of the poor $M I$ 's behavior, conditions for such coordination are most favorable when the poorest

\footnotetext{
${ }^{28}$ Fifty-seven percent of $M J$ groups with a propensity to coordinate on $P$ in the group appeal treatment have a negative and significant time trend in their support of candidate $R$ but in no $M J$ groups with a propensity to coordinate on $R$ do we observe a significantly increasing vote share of candidate $R$ over time. These time trends in support of candidate $P$ and $R$ are the coefficient estimates taken from a regression of the average vote share of $R$ on round of play run on observations from $M J$ in each decision group separately.
} 
member is very poor and most likely to vote for the redistributive candidate $P$ (assigned income of 10, 22, or 27 with medium and high group heterogeneity) instead of moderately poor (assigned income of 44 with low group heterogeneity). Indeed, the average rate of votes for candidate $P$ in $M J$ is significantly higher in the former than the latter (difference is .34). ${ }^{29}$

Result 3 Group appeals trigger equilibrium coordination on the candidate who imposes costs on most members of their own group in individual-level benefit by making voters weigh their expectations about the behavior of fellow group members and of members of the other group more highly. Groupmajoritarian coordination as decision heuristic arises only rarely.

Note: an alternative mechanism to group-majoritarian coordination, similar to the one behind equilibrium coordination, may better explain why $M J$ members often vote for candidate $R$ despite such behavior being incongruent with an equilibrium profile: vote choice is determined by $M J$ 's expectations about the behavior of its poorest member. The willingness of the poorest member of $M J$ to coordinate with richer fellow members and these richer members' belief about the poorest member's willingness to coordinate is largest when the group-level benefit offsets the loss in the individual-level benefit (i.e., when group heterogeneity is low). Indeed, the richer members of $M J$ are by .30 significantly more likely to vote for candidate $R$ when group heterogeneity in $M J$ is low than when it is medium or high.

\subsection{Robustness of experimental results}

To assess the experimental results presented, I investigate the possibility of learning effects and whether the finding of equilibrium coordination is robust to an alternative other-regarding preferences mechanism.

Subjects interact with others in fixed societies over several rounds. Any behavior observed and any treatment effect identified may result from or vary with learning. Averaging appeal treatments in elections where group heterogeneity of $M J$ is low shows that the relative frequency of the $(R, R, R ; P, P)$ strategy profile increases significantly in the final third of the experiment over the first and second thirds. While that relative frequency is .33 in round 1-12 and .29 in round 13-24, it

\footnotetext{
${ }^{29}$ Exit-survey responses also indicate that $M J$ with a propensity to coordinate on $P$ are more likely than $M J$ with a propensity to coordinate on $R$ to consider whether others are affected by the group appeal. $42 \%$ of subjects in $M J$ with a propensity to coordinate on $P$ report that they believed appeals affected not only themselves, but also others, while only $33 \%$ did so in $M J$ with a propensity to coordinate on $R$.
} 
rises significantly to .53 in round $25-36 .^{30}$ The equilibrium coordination mechanism, given the time trend in voters' choices, surely indicates such learning effects as well. Realizing that such learning effects only arise for societies for which I can reasonably claim they follow equilibrium coordination but not for those who may follow group-majoritarian coordination, makes the observation of learning effects part of the evidence for equilibrium coordination being an instance of sophisticated decision-making and not an issue of a lack of robustness.

The effect of group appeals that trigger equilibrium coordination on the redistribution candidate $P$ may be confounded by yet another mechanism: heightened other-regarding preferences in the form of increased inequity aversion (Fehr and Schmidt, 1999). If this effect of group appeal exists, it could arise from experimenter demand effects (i.e., social desirability). Convergence on $P$ of $M I$ and those $M J$ groups that follow equilibrium coordination could be a sign of a stronger preference for redistribution emerging with the group appeal (including concern for the identity group's poorest members). To show that there is no such effect, I reverse the income distribution creating a mostly poor $M J$ and a rich $M I$. Now, the target of equilibrium coordination in $M J$ is shifted to the wealth-preserving candidate $R$, and we should see effects of the group appeal accordingly. Conversely, if preferences for redistribution are behind the effects of group appeals on coordination, the group appeal should still lead to an increase in vote share of $P$. Overall, when reversing the income distribution, the vote share of candidate $R$ increases significantly. While it was .38 in no appeal and group appeal treatments with a mostly rich $M J$, it is now .71 in the Poor $M J$-No appeal treatment and .61 in the Poor $M J$-Group appeal treatment.

\section{Discussion and conclusion}

In this study, I demonstrate how groups coordinate in elections to win group-targeted benefits. With increasing group heterogeneity, the candidate who is individually-beneficial to most members of the group loses support. This re-alignment partially arises because group appeals raise the awareness of voters to how their fellow group members might act and how other groups behave. Why do we observe this mechanism? Voting is characterized by the opportunity to secure grouptargeted benefits but individuals are required to make reasonable guesses about the responses of

\footnotetext{
${ }^{30}$ See Figure C.6 in the online Appendix for the distribution of strategy profiles played in the first, second, and final third of the experiment.
} 
others to secure them. Individuals rely on focal points in decision situations that contain uncertainty. Group markers generate common knowledge about such focal points among the members of a group allowing for successful coordination similar to other more context-rich public signals, such as rituals (Chwe, 2013), symbols (Schnakenberg, 2014), or information about political outcomes (Mebane and Sekhon, 2002).

Group-majoritarian coordination would provide such a simple cue - maximize group welfare to rally the group around an alternative by parameterizing a strategic problem in which individuals maximizing group utility are guided towards a clear coordination target (Guala, Mittone and Ploner, 2013). Here, group identity serves as a low-cost informational cue in complex decision-making environments (Lupia and McCubbins, 1998) and is most valuable to individuals who are otherwise less informed (Bassi, Morton and Williams, 2011).

The uncertainty about how other groups will behave, however, seems sufficient to steer voters away from this easily recognizable focal point. A group rationality now must emerge so the group finds an optimal strategy vis-a-vis the other group; that is more easily done when the group is appealed to (Bornstein, Gneezy and Nagel, 2002). As an instance of such rationality, decision makers follow a mechanism - equilibrium coordination - by which joint group action arises from far less obvious considerations of the preferences and expected behaviors of fellow group members and the other group.

What exactly is behind equilibrium coordination? While the observed electoral outcomes in the experiment result from features of the game I implement, the mechanism by which subjects arrive at them is driven by a more fundamental response to a group appeal: decision makers become increasingly aware of the expectations of others, strategic uncertainties resolve and, ultimately, coordinated collective behavior of a group emerges. ${ }^{31}$ Group identities help to create common conjecture; they serve to persuade the individual that others will take a specific action, that others are similarly convinced that everyone else will also take this action, that others are certain that the individual will take this action, etc. My study provides clear evidence of the existence of common conjecture with group identities by a counterfactual: with income appeals the content of the common conjecture is muted and the resulting coordination changes with a different income

\footnotetext{
${ }^{31}$ Strategic uncertainty arises when the rational decision maker deductively formulates beliefs about the state of the world and others' behavior but does not know for sure which equilibrium concept other decision makers will use (Van Huyck, Battalio and Beil, 1990).
} 
distribution where the target of the group's joint action is shifted.

The 1997 mayoral election in Los Angeles, in which Republican businessman Richard Riordan was pitted against State Senator Democrat Tom Hayden, is a telling empirical anecdote illustrating equilibrium coordination. In their campaigns, both appealed heavily to Latino voters for their support by offering group-targeted benefits. Riordan pushed for massive transfers to Los Angeles's schools (Kaufmann, 2003, 162), which are dominated by Latino students, and Hayden took a strong stance against anti-illegal immigration initiatives (Newton, 1997). Despite the fact that only $43 \%$ of Latino voters supported Riordan in the previous mayoral race, on the election day in 1997, according to Los Angeles Times exit polls, he scored $60 \%$ of the Latino vote (Kaufmann, 2003, 164) in a city where Democrats outnumber Republicans two-to-one. His success with Latino voters was largely attributed to his ability to convince them that he would continue to strengthen the public education system (Kaufmann, 2003, 162) even though he may promote economic policies that would be to the detriment of many members of that sub-population, who are "more likely to be working class" (Sonenshein, 2004, 95). While many features of this particular race may determine why so many Latinos voted for Riordan, observers were surprised by how individual voters traded off individuallevel benefits implied by the candidates' position on redistribution against group-targeted benefits implied by their position on education. One may have expected Latinos to coordinate on voting for the candidate offering group-targeted benefits but whose redistributive policies are also most beneficial for the majority of group members. Such group-majoritarian coordination did not occur.

For another more recent example of the existence of the described rationale, consider the runup to the 2012 presidential election where President Obama's campaign grew concerned about the potential for a "huge white turnout" (Warren, 2012) and Republicans complained about the supposedly automatic support of minorities for the incumbent President. ${ }^{32}$ In a race-salient election, everyone was aware of the fact that the opposing candidate may do a better job than usual in mobilizing in-group voters. This concern generates an even greater willingness to turn out for the co-racial candidate and is a perfectly reasonable strategic response to the salience of race, going well beyond electoral support driven by emotional attachment or shared interests. A speculative observation suggests that the Obama campaign may have been reluctant to openly appeal to minority

\footnotetext{
${ }^{32}$ As a racial minority, Obama certainly engenders emotional attachment among African Americans and potentially other minorities, and his platform speaks more to the concerns of minorities than to the white majority of Americans.
} 
voters as it could serve to raise awareness among the racial majority of the potential for a large minority turnout. One could argue that the fear of group-majoritarian coordination, a division of the electorate along race lines, led the Obama campaign to try to not further appeal to voters based on their race group.

Ethnic politics provides further interesting applications. As example for how a group appeal triggers a coordination mechanism that seems unexpected at first, Wilkinson (2004) shows that group appeals to the Muslim minority in India are a viable tool to add non-Hindu voters to a larger Hindu-based electoral coalition because it also positively affects the willingness of the Hindu majority to bandwagon and vote for the minority-appealing candidate.

Generally, my study shows that even weak, context-free identities, as implemented in the laboratory, affect individuals' beliefs and behaviors in group-driven political competition similar to what has been found outside of the laboratory (e.g., Michelitch (2015); Eifert, Miguel and Posner (2010)). The group appeal treatment effect however, mostly speaks to effects on individuals' beliefs. In my account, convergence of members of one social group on a particular electoral alternative should be seen as information-driven herding (Goeree and Yariv, 2015) or as bandwagoning (Bartels, 1988), and not so much as a result of a saliency-triggered shift from personal to collective identity and an increased adherence to group norms (Huddy, 2013). Nevertheless, the mechanism I identify differs from bandwagoning because it does not need the sequential nature of voting and the information provided in such a process for voters to engage in it (Morton and Williams, 1999; Morton and Ou, 2015).

Even more generally, I establish the existence of a coordination mechanism in the context of elections where voters respond to a call for coordinated group action by changing their behavior in response to how they expect the appeal to shape the actions of others. Indeed, such group appeal does not necessarily lead an individual to adhere to group norms or to value the overall welfare of the group. It makes it more likely that the voter considers the expected behavior of others and how everyone else might respond to the voters choice. Group identity primed in such way hastens coordination on the candidate most likely to be the electoral victor, leading some voters to abandon their "natural" electoral alternative given their preferences over individual-level benefits. Often, though, the intuitive effect to divide society along group lines persists even though those divisions may, as I show here, emerge from a strategic rationale. 


\section{References}

Akerlof, George and Rachel Kranton. 2000. "Economics and Identity." Quarterly Journal of Economics 115(3):715-53.

Andreoni, James. 1989. "Giving with Impure Altruism: Applications to Charity and Ricardian Equivalence." Journal of Political Economy 97(6):1447-58.

Ashworth, Scott and Kenneth W Shotts. 2014. "Challengers and Electoral Accountability.".

Bartels, Larry. 1988. Presidential Primaries and the Dynamics of Public Choice. Princeton: Princeton University Press.

Bassi, Anna, Rebecca B Morton and Kenneth C Williams. 2011. "The effects of identities, incentives, and information on voting." The Journal of Politics 73(2):558-571.

Bernhard, Helen, Ernst Fehr and Urs Fischbacher. 2006. "Group Affiliation and Altruistic Norm Enforcement." American Economic Review 96(2):217-21.

Bernheim, D. 1994. "A Theory of Conformity." Journal of Political Economy 102(5):842-77.

Bornstein, Gary. 2003. "Intergroup conflict: Individual, group, and collective interests." Personality and social psychology review 7(2):129-145.

Bornstein, Gary, Uri Gneezy and Rosmarie Nagel. 2002. "The effect of intergroup competition on group coordination: An experimental study." Games and Economic Behavior 41(1):1-25.

Carlson, Elizabeth. 2015. "Ethnic voting and accountability in Africa: A choice experiment in Uganda." World Politics 67(2):353-385.

Chandra, Kanchan. 2004. Why Ethnic Parties Succeed. New York: Cambridge University Press.

Chen, Yan and Sherry Li. 2009. "Group Identity and Social Preferences." American Economic Review 99(1):431-57.

Chwe, Michael Suk-Young. 2013. Rational ritual: Culture, coordination, and common knowledge. Princeton University Press.

Dahl, Robert A. 2005. Who governs?: Democracy and power in an American city. Yale University Press.

Dickson, Eric and Kenneth Scheve. 2006. "Social Identity, Political Speech, and Electoral Competition." Journal of Theoretical Politics 18(1):5-39.

Eckel, Catherine and Philip Grossman. 2005. "Managing Diversity by Creating Team Identity." Journal of Economic Behavior \& Organization 58:371-392.

Eifert, Benn, Edward Miguel and Daniel N Posner. 2010. "Political competition and ethnic identification in Africa." American Journal of Political Science 54(2):494-510.

Fehr, Ernst and Klaus Schmidt. 1999. "A Theory of Fairness, Competition, and Cooperation." Quarterly Journal of Economics 114(3):817-68.

Ferree, Karen E. 2006. "Explaining South Africa's racial census." Journal of Politics 68(4):803-815. 
Ferree, Karen and Jeremy Horowitz. 2010. "Ties that bind? The rise and decline of ethno-regional partisanship in Malawi, 1994-2009." Democratization 17(3):534-563.

Fryer, Roland G, Jacob K Goeree and Charles A Holt. 2005. "Experience-based discrimination: Classroom games." The Journal of Economic Education 36(2):160-170.

Goeree, Jacob K and Leeat Yariv. 2015. "Conformity in the lab." Journal of the Economic Science Association 1(1):15-28.

Goette, Lorenz, David Huffman and Stephan Meier. 2006. "The Impact of Group Membership on Cooperation and Norm Enforcement: Evidence Using Random Assignment to Real Social Groups." American Economic Review 96(2):212-6.

Gordon, Sanford, Gregory Huber and Dimitri Landa. 2007. "Challenger Entry and Voter Learning." American Political Science Review 101(2):303.

Gottlieb, Jessica, Guy Grossman, Horacio Larreguy and Benjamin Marx. 2019. "A Signaling Theory of Distributive Policy Choice: Evidence from Senegal." The Journal of Politics 81(2):631-647.

Guala, Francesco, Luigi Mittone and Matteo Ploner. 2013. "Group membership, team preferences, and expectations." Journal of Economic Behavior \& Organization 86:183-190.

Harding, Robin. 2015. "Attribution and accountability: Voting for roads in Ghana." World Politics 67(4):656-689.

Hogg, Michael A. 1996. "Intragroup processes, group structure and social identity." Social groups and identities: Developing the legacy of Henri Tajfel 65:93.

Huddy, Leonie. 2001. "From Social to Political Identity: A Critical Examination of Social Identity Theory." Political Psychology 22(1):127-56.

Huddy, Leonie. 2013. From Group Identity to Political Cohesion and Commitment. In The Oxford Handbook of Political Psychology. Oxford University Press pp. 737-73.

Ichino, Nahomi and Noah L Nathan. 2013. "Crossing the line: Local ethnic geography and voting in Ghana." American Political Science Review 107(2):344-361.

Kaufmann, KM. 2003. The mayoral politics of New York and Los Angeles. In New York and Los Angeles: Politics, Society, and Culture-A Comparative View. pp. 314-340.

Kitschelt, Herbert and Steven I Wilkinson. 2007. Patrons, clients and policies: Patterns of democratic accountability and political competition. Cambridge University Press.

Klor, Esteban and Moses Shayo. 2010. "Social Identity and Preferences over Redistribution." Journal of Public Economics 94(3):269-78.

Landa, Dimitri and Dominik Duell. 2015. "Social Identity and Electoral Accountability." American Journal of Political Science 59(3):671-89.

Lupia, Arthur and Mathew McCubbins. 1998. The Democratic Dilemma: Can citizens Learn What They Need to Know? Cambridge: Cambridge University Press.

Mebane, Walter R and Jasjeet S Sekhon. 2002. "Coordination and policy moderation at midterm." American Political Science Review 96(01):141-157. 
Michelitch, Kristin. 2015. "Does electoral competition exacerbate interethnic or interpartisan economic discrimination? Evidence from a field experiment in market price bargaining." American Political Science Review 109(1):43-61.

Morton, Rebecca B. 1991. "Groups in rational turnout models." American Journal of Political Science pp. $758-776$.

Morton, Rebecca B and Kai Ou. 2015. "What motivates bandwagon voting behavior: Altruism or a desire to win?" European Journal of Political Economy 40:224-241.

Morton, Rebecca and Kenneth Williams. 1999. "Information Asymmetries and Simultaneous versus Sequential Voting." American Journal of Political Science 93(1):51-67.

Newton, Jim. 1997. "Mayor's Race Focuses Intently on Latino Vote.".

URL: http://articles.latimes.com/1997-03-05/news/mn-35033_1_latino-voters

Posner, Daniel. 2005. Institutions and Ethnic Politics in Africa. Cambridge: Cambridge University Press.

Roemer, John. 1998. "Why the Poor Do Not Expropriate the Rich: An Old Argument in New Garb." Journal of Public Economics 70(399-424).

Scheve, Kenneth and David Stasavage. 2006. "Religion and Preferences for Social Insurance." Quarterly Journal of Political Science 1:255-86.

Schnakenberg, Keith E. 2014. "Group Identity and Symbolic Political Behavior." Quarterly Journal of Political Science 9:137-167.

Schram, Arthur and Joep Sonnemans. 1996. "Why People Vote: Experimental Evidence." Journal of Economic Psychology 17(4):417-42.

Sen, Maya and Omar Wasow. 2016. "Race as a bundle of sticks: Designs that estimate effects of seemingly immutable characteristics." Annual Review of Political Science 19:499-522.

Shayo, Moses. 2009. "A Model of Social Identity with an Application to Political Economy: Nation, Class, and Redistribution." American Political Science Review 103(2):147-74.

Smith, Alastair and Bruce Bueno De Mesquita. 2012. "Contingent prize allocation and pivotal voting." British Journal of Political Science 42(02):371-392.

Sonenshein, Raphael J. 2004. The city at stake: Secession, reform, and the battle for Los Angeles. Princeton University Press.

Stokes, Susan C. 2005. "Perverse accountability: A formal model of machine politics with evidence from Argentina." American Political Science Review 99(3):315.

Suhay, Elizabeth. 2015. "Explaining group influence: The role of identity and emotion in political conformity and polarization." Political Behavior 37(1):221-251.

Tajfel, Henri. 1981. Human Groups and Social Categories. Cambridge: Cambridge University Press.

Tajfel, Henri and John Turner. 1986. The Social Identity Theory of Intergroup Behavior. In The Psychology of Intergroup Relations, ed. Stephen Worchel and William Austin. Chicago: NelsonHall pp. 7-24. 
Tajfel, Henri and Michael Billig. 1974. "Familiarity and Categorization in Intergroup Behavior." Journal of Experimental Social Psychology 10:159-70.

Van Huyck, John, Raymond Battalio and Richard Beil. 1990. "Tacit Coordination Games, Strategic Uncertainty, and Coordination Failure." American Economic Review 80(1):234-48.

Warren, James. 2012. "Team Obama's Election Day Anxiety: Fear of a Big White Vote.".

URL: http://www.thedailybeast.com/articles/2012/11/06/team-obama-s-election-day-anxietyfear-of-a-big-white-vote.html

Weghorst, Keith R and Staffan I Lindberg. 2013. "What drives the swing voter in Africa?" American Journal of Political Science 57(3):717-734.

White, Ismail K, Chryl Laird and Troy D Allen. 2014. "Selling Out?: The Politics of Navigating Conflicts Between Racial Group Interest and Self-Interest." American Political Science Review 108(04):783-800.

Wilkinson, Steven. 2004. Votes and Violence: Electoral Competition and Ethnic Riots in India. Cambridge: Cambridge University Press. 


\section{Online appendix}

\section{A Theoretical appendix}

\section{A.1 Proofs}

I begin the analysis with the following claim, which shows that a strategy profile where any member of $M I$ plays $R$ cannot be an equilibrium:

Claim 1 There exists no equilibrium in which some $i \in M I$ choose $R$.

Proof Consider all strategy profiles where $i \in M I$ is pivotal: such profiles are of the kind that $i$ either (i) is decisive in the election between $P$ and $R$, (ii) is decisive in securing $\mathcal{I}$ fully from candidate $P$, (iii) is decisive in securing $1 / 2$ of $\mathcal{I}$ from candidate $P$ (shared with $M J$ ), or (iv) is decisive in securing $1 / 2$ of $\mathcal{I}$ from candidate $R$ (shared with $M J$ ). In strategy profiles (i)-(iii), $i$ strictly prefers choosing $P$ over $R$. The strategy profile representing (iv) is of the form $\left(P, R, R ; R, \alpha_{i}\right)$. Given this profile, $\alpha_{i}$ assigning probability 1 to $a_{i}=R$ yields a profile that is not sustainable in equilibrium because $j \in M J$ playing $P$ has an incentive to deviate to $R$ to gain the full $\mathcal{I}$ for $M J$ from candidate $R$. Thus, a member of $M I$ choosing $R$ cannot be an equilibrium. By the assumption that $U_{i}^{C}$ determines vote choice when $i$ is indifferent, $i$ chooses $P$ because $\omega_{i}<\omega_{M}$ for all $i \in M I$ in all strategy profiles where $i$ is not pivotal.

Equipped with the equilibrium prediction about $M I$ choosing $P$ in Claim $1, \mathrm{I}$ arrive at the main proposition.

\section{Proof of Proposition 1}

To see that $(P, P, P ; P, P)$ is an equilibrium, suppose that one voter in $M J$ deviates and votes for the other candidate. Then, her group will need to share the group benefit with $M I$ because the winning candidate would now be supported by two voters from each group and that will mean a drop in her expected utility, making this deviation unprofitable. Holding everybody else fixed, no member of $M I$ has a profitable deviation given that the voting outcome is fully determined by the unanimous vote of members of $M J$ and those members capture the group-level benefit.

To see that $(P, P, P ; P, P)$ is also the unique pure strategy equilibrium in which candidate $P$ wins the election ( $P$-equilibrium), note first that by Claim 1, only strategy profiles where all members of $M I$ choose $P$ can be an equilibrium profile. This leaves only profiles $(R, P, P ; P, P)$ and $(R, R, P ; P, P)$ as other candidates for a $P$-equilibrium. Neither profile can be an equilibrium, however, because $i \in M J$ choosing $R$ has an incentive to deviate to $P$ to secure $\mathcal{I}$ from candidate $P$ in the former strategy profile and to secure sharing $\mathcal{I}$ from candidate $P$ with $M I$ in the latter profile.

To see that $(R, R, R ; P, P)$ is an equilibrium ( $R$-equilibrium), suppose members of $M J$ vote for $R$ and members of $M I$ vote for $P$. Solving for $\omega_{i}$ reveals that no member of $M J$ is willing to deviate to $P$ as long as $\omega_{i}>(V-I) / \tau=\omega^{L}$. By Claim 1, and because the voting outcome is fully determined by the unanimous vote of members of $M J$ capturing the group-level benefit $I$, no member of $M I$ has a profitable deviation.

Given uniqueness when the poorest member of $M J$ is very poor, an equilibrium in mixed strategies exist if and only if all members of $M J$ are not very poor, i.e. if their incomes are higher than $\omega_{L}=\frac{V-I}{\tau}$. To derive the mixed strategy equilibrium, let $E U_{1}(P)$ and $E U_{1}(R)$ be the expected utility of player 1 who is a member of $M J$ from playing $P$ and $R$, respectively. Further, let $p_{2}$ and $p_{3}$ be the probabilities that the other two members of $M J$, player 2 and player 3, play $P$, respectively, 
and recall that by Claim 1, the two members of $M I$ play $P$ with probability 1 . Therefore $E U_{1}(P)$ and $E U_{1}(R)$ are given by

$$
\begin{aligned}
& E U_{1}(P)=\left(U_{1}^{P}+\mathcal{I}\right) p_{2} p_{3}+\left(U_{1}^{P}+\frac{\mathcal{I}}{2}\right)\left[p_{2}\left(1-p_{3}\right)+\left(1-p_{2}\right) p_{3}\right]+U_{1}^{P}\left(1-p_{2}\right)\left(1-p_{3}\right) \\
& E U_{1}(R)=\left(U_{1}^{P}+\frac{\mathcal{I}}{2}\right) p_{2} p_{3}+U_{1}^{P}\left[p_{2}\left(1-p_{3}\right)+\left(1-p_{2}\right) p_{3}\right]+\left(U_{1}^{R}+\mathcal{I}\right)\left(1-p_{2}\right)\left(1-p_{3}\right)
\end{aligned}
$$

In order for player 1 to randomize it has to be that she is indifferent between playing $\mathrm{P}$ and $\mathrm{R}$, i.e. that:

$$
\begin{gathered}
E U_{1}(P)=\left(U_{1}^{P}+\mathcal{I}\right) p_{2} p_{3}+\left(U_{1}^{P}+\frac{\mathcal{I}}{2}\right)\left[p_{2}\left(1-p_{3}\right)+\left(1-p_{2}\right) p_{3}\right]+U_{1}^{P}\left(1-p_{2}\right)\left(1-p_{3}\right)= \\
\left(U_{1}^{P}+\frac{\mathcal{I}}{2}\right) p_{2} p_{3}+U_{1}^{P}\left[p_{2}\left(1-p_{3}\right)+\left(1-p_{2}\right) p_{3}\right]+\left(U_{1}^{R}+\mathcal{I}\right)\left(1-p_{2}\right)\left(1-p_{3}\right)=E U_{i}(R)
\end{gathered}
$$

Simplifying gives us the indifference condition

$$
\left(U_{1}^{P}-U_{1}^{R}\right)\left(1-p_{2}\right)\left(1-p_{3}\right)+\mathcal{I}\left[\frac{3}{2}\left(p_{2}+p_{3}\right)-p_{2} p_{3}-1\right]=0
$$

In equilibrium, player 2 must mix with probability

$$
p_{2}^{*}=\frac{\left(U_{1}^{P}-U_{1}^{R}\right)\left(1-p_{3}\right)-\mathcal{I}\left(1-3 / 2 p_{3}\right)}{\left(U_{1}^{P}-U_{1}^{R}\right)\left(1-p_{3}\right)-\mathcal{I}\left(3 / 2-p_{3}\right)}
$$

and player 3 with probability

$$
p_{3}^{*}=\frac{\left(U_{1}^{P}-U_{1}^{R}\right)\left(1-p_{2}\right)-\mathcal{I}\left(1-3 / 2 p_{2}\right)}{\left(U_{1}^{P}-U_{1}^{R}\right)\left(1-p_{2}\right)-\mathcal{I}\left(3 / 2-p_{2}\right)}
$$

Following similar steps, we can show that in equilibrium player 1 must mix with probability

$$
p_{1}^{*}=\frac{\left(U_{2}^{P}-U_{2}^{R}\right)\left(1-p_{3}\right)-\mathcal{I}\left(1-3 / 2 p_{3}\right)}{\left(U_{2}^{P}-U_{2}^{R}\right)\left(1-p_{3}\right)-\mathcal{I}\left(3 / 2-p_{3}\right)}
$$

The probabilities of playing $P$ for players $i=\{1,2,3\} \in M J,\left(\alpha_{1}^{* M J}(P), \alpha_{2}^{* M J}(P), \alpha_{3}^{* M J}(P)\right)$, for the equilibrium strategy $\alpha_{i}^{* G}$, then, are the solution to the system:

$$
\left\{\begin{array}{l}
p_{1}=\frac{\delta U_{2}\left(1-p_{3}\right)-\mathcal{I}\left(1-3 / 2 p_{3}\right)}{\delta U_{2}\left(1-p_{3}\right)-\mathcal{I}\left(3 / 2-p_{3}\right)} \\
p_{2}=\frac{\delta U_{1}\left(1-p_{3}\right)-\mathcal{I}\left(1-3 / 2 p_{3}\right)}{\delta U_{1}\left(1-p_{3}\right)-\mathcal{I}\left(3 / 2-p_{3}\right)} \\
p_{3}=\frac{\delta U_{1}\left(1-p_{2}\right)-\mathcal{I}\left(1-3 / 2 p_{2}\right)}{\delta U_{1}\left(1-p_{2}\right)-\mathcal{I}\left(3 / 2-p_{2}\right)}
\end{array}\right.
$$

with $p_{i}=\alpha_{i}^{* M J}(P)$ and $\delta U_{i}=U_{i}^{P}-U_{i}^{R}$ for $i \in M J$. 


\section{A.2 Extensions}

Consider a model where the distribution of the individual-level attribute income is not contingent on group identity. For this game, I will restrict analysis to the pure strategy Nash equilibria. Equilibrium strategy profiles of this game are of the form $\left(a_{1}^{\mathrm{MJ}}, a_{2}^{\mathrm{MJ}}, a_{3}^{\mathrm{MJ}}, a_{1}^{\mathrm{MI}}, a_{2}^{\mathrm{MI}}\right)$ where $a_{i}^{\mathrm{MJ}}$, $i=\{1,2,3\}$, are the pure strategies chosen by the three members of $M J$ and $a_{j}^{\mathrm{MI}}, j=\{1,2\}$, are the pure strategies chosen by the two members of $M I$.

To see that the profiles $(P, P, P ; P, P)$ and $(R, R, R ; R, R)$ are Nash equilibria in pure strategies, suppose one voter in $M J$ deviates and votes for the other candidate. Then, her group will need to share the group benefit with $M I$ because the winning candidate would now be supported by two voters from each group and that will mean a drop in her expected utility, making this deviation unprofitable. Holding everybody else fixed, no member of $M I$ has a profitable deviation given that the voting outcome is fully determined by the unanimous vote of members of $M J$ and those members capture the group-level benefit. Note, for the same reason, the strategy profiles $(P, P, P ; P, R)$, $(P, P, P ; R, P),(R, R, R ; P, R)$, and $(R, R, R ; R, P)$ are also Nash equilibria in pure strategies.

The following proposition characterizes another $R$-equilibrium and another $P$-equilibrium whose existence is income dependent.

Proposition 2 An equilibrium exists where all members of $M J$ vote for $P$ if they are not very rich, i.e. if their incomes are lower than $\omega^{H}=\frac{V+I}{\tau}$, or $R$ if they are not very poor, i.e. if their incomes are higher than $\omega^{L}=\frac{V-I}{\tau}$, while all members of $M I$ vote for $R$ and $P$, respectively.

Strategy profiles fitting the description of income-dependent equilibria are (1) $\forall j \in M J$ s.th. $w_{j} \leq$ $\omega_{H}$ and $\forall i \in M I,(P, P, P ; R, R)$ and (2) $\forall j \in M J$ s.th. $w_{j} \geq \omega_{L}$ and $\forall i \in M I,(R, R, R ; P, P)$.

Proof To see why (1) is an equilibrium, suppose members of $M J$ vote for $P$ and members of $M I$ vote for $R$. Considering a deviation, a member of $M J$ trades off receiving a payoff of $(1-\tau) \omega_{i}+V+I$ from voting with her fellow group members and $\omega_{i}$ from voting with the other group. Solving for $\omega_{i}$ reveals that any member of $M J$ is willing to vote for $P$ as long as $\omega_{i}<(V+I) / \tau=\omega^{H}$. Equivalently, to see why (2) is an equilibrium suppose members of $M J$ vote for $R$ and members of $M I$ vote for $P$. Solving for $\omega_{i}$ reveals that any member of $M J$ is willing to vote for $R$ as long as $\omega_{i}>(V-I) / \tau=\omega^{L}$. Holding the actions of everybody else fixed, no member of $M I$, again, has a profitable deviation given that the voting outcome is fully determined by the unanimous vote of members of $M J$ and those members capture the group-level benefit.

There are three sets of strategy profiles not characterized so far; all of these profiles are not a Nash equilibrium in pure strategies. To see why this statement is true, first, consider profiles where both members of $\mathrm{MI}$ and one member of $M J$ vote for the same alternative. These are the profiles $(P, P, R ; R, R),(P, R, P ; R, R),(R, P, P ; R, R),(R, R, P ; P, P),(R, P, R ; P, P)$, and $(P, R, R ; P, P)$. Here any of the two other members of $M J$ who voted for the other alternative have an incentive to deviate to secure to share $\mathcal{I}$ with the members of MI; otherwise members of MI would enjoy $\mathcal{I}$ exclusively. Second, consider profiles where both members of MI and two members of $M J$ vote for the same alternative. These are the profiles $(P, R, R ; R, R),(R, R, P ; R, R)$, $(R, P, R ; R, R),(R, P, P ; P, P),(P, P, R ; P, P)$, and $(P, R, P ; P, P)$. Here the other member of $M J$ who voted for the other alternative has an incentive to deviate to secure $\mathcal{I}$ for $M J$ exclusively instead of sharing it with the members of $M I$. Third, consider any profile where members of MI are evenly split over alternatives $P$ and $R$ and members of $M J$ split one-to-two. These are the profiles $(P, P, R ; P, R),(R, P, P ; P, R),(P, R, P ; P, R),(P, P, R ; R, P),(R, P, P ; R, P),(P, R, P ; R, P)$, $(R, R, P ; P, R),(P, R, R ; P, R),(R, P, R ; P, R),(R, R, P ; R, P),(P, R, R ; R, P)$, and $(R, P, R ; R, P)$. For such profiles the member of $M I$ who is not voting for the winning alternative has an incentive to deviate to secure for MI sharing $\mathcal{I}$ with $M J$; otherwise members of $M J$ would enjoy $\mathcal{I}$ exclusively. 


\section{B Experimental design appendix}

\section{B.1 Experimental sessions}

Experimental sessions were carried out in an experimental social science lab at Technical University Berlin. Participants signed up via a web-based recruitment system, ORSEE (Greiner, 2015), that draws on a large, pre-existing pool of potential subjects. Subjects were not recruited from the author's courses. The recruitment system contains a filter that blocked subjects from participating in more than one session of a given experiment. The subject pool consists almost entirely of students from around the university.

Subjects interacted anonymously via networked computers. The experiments were programmed and conducted with the software z-Tree (Fischbacher, 2007). After giving informed consent according to standard human subjects protocols, subjects received written instructions that were subsequently read aloud in order to promote understanding and induce common knowledge of the experimental protocol. In accordance with the long-standing norms of the lab in which the experiment was carried out, no deception was employed at any point in the experiment. Before the voting game stage commenced, subjects were asked three questions concerning their understanding of the payoff tables provided to them in the instructions. $90 \%$ of participating subjects answered those questions correctly. At the end of the experiment, an exit survey was conducted. Subjects received a show-up fee of $\$ 7$ (5 Euro) and performance-based payments of on average $\$ 22$ (16 Euro) for an experiment that lasted about 1 hour. Payments from the voting game where taken from the higher round-payoff from two randomly selected rounds.

\section{B.2 Group identity inducement stage}

To induce identities subjects were shown 5 pairings of paintings, one by Paul Klee and one by Vassily Kandinsky, and were asked to choose their preferred painting in each pair. Based on which painter's work a subject prefers most of the time, he or she was assigned to be a Klee or a Kandinsky and subjects engaged in a collaborative quiz within their painter identity group.

\section{B.3 Treatments}

For robustness checks, I implement a series of supplemental treatments: I repeat treatments that resemble no appeal, group appeal, and income appeal treatments now with a mostly poor $M J$ and a mostly rich overall society (Poor $M J$-No appeal, Poor $M J$-Group appeal, and Poor $M J$-Income appeal), and the group appeal treatment again but now with all members of $M I$ assigned a high income (Rich $M I$-Group appeal treatment). The Poor $M J$ treatments include 12 rounds of the low group heterogeneity treatment (instead of just 8) but only 24 rounds of the medium group heterogeneity treatment. The Rich $M I$-Group appeal treatment is played for 30 rounds only: 10 rounds with low group heterogeneity and 20 rounds with medium heterogeneity. Across the seven treatments, I collect 13500 subject-round observations on 340 subjects in 68 societies. 
Table B.2: Summary of all treatment conditions and treatment statistics.

\begin{tabular}{|c|c|c|c|c|c|c|c|}
\hline \multicolumn{2}{|c|}{ Appeal treatments } & \multirow[t]{3}{*}{ Societies } & \multirow[t]{3}{*}{ Subjects } & \multicolumn{4}{|c|}{ Subject-round observations } \\
\hline & & & & \multicolumn{4}{|c|}{ by level of group heterogeneity } \\
\hline & & & & Total & Low & Medium & High \\
\hline $\begin{array}{r}\text { Main } \\
\text { treatments }\end{array}$ & $\begin{array}{r}\text { No appeal } \\
\text { Group appeal } \\
\text { Income appeal }\end{array}$ & $\begin{array}{c}14 \\
16 \\
8\end{array}$ & $\begin{array}{l}70 \\
80 \\
40\end{array}$ & $\begin{array}{c}\text { (40 rounds) } \\
2800 \\
3200 \\
1600\end{array}$ & $\begin{array}{c}\text { (8 rounds) } \\
560 \\
640 \\
320\end{array}$ & $\begin{array}{c}\text { (28 rounds) } \\
1960 \\
2240 \\
1120\end{array}$ & $\begin{array}{l}\text { (4 rounds) } \\
280 \\
320 \\
160\end{array}$ \\
\hline Supplemental & $\begin{array}{r}\text { Poor } M J \text {-No appeal } \\
\text { Poor } M J \text {-Group appeal } \\
\text { Poor } M J \text {-Income appeal }\end{array}$ & $\begin{array}{c}9 \\
11 \\
8\end{array}$ & $\begin{array}{l}45 \\
55 \\
40\end{array}$ & $\begin{array}{c}\text { (40 rounds) } \\
1800 \\
2200 \\
1600\end{array}$ & $\begin{array}{c}\text { (12 rounds) } \\
540 \\
660 \\
480\end{array}$ & $\begin{array}{c}\text { (24 rounds) } \\
1080 \\
1320 \\
960\end{array}$ & $\begin{array}{l}\text { (4 rounds) } \\
180 \\
220 \\
160\end{array}$ \\
\hline & Rich $M I$-Group appeal & 2 & 10 & $\begin{array}{c}(30 \text { rounds }) \\
300\end{array}$ & $\begin{array}{c}\text { (10 rounds) } \\
100\end{array}$ & $\begin{array}{c}\text { (20 rounds) } \\
200\end{array}$ & - \\
\hline Total & & 68 & 340 & 13500 & 3300 & 8880 & 1320 \\
\hline
\end{tabular}

There is balance in treatment conditions compared to the no appeals treatment of the rich $M J$ treatments. The distributions of a variable that records subjects' "closeness" to their identity group are indistinguishable across conditions (See Table B.3). Out of the five comparisons between treatment condition and no appeal treatment over seven balance variables (age, Germans origin, attitudes towards welfare state, attitudes towards being taxed for increasing education spending, attitudes towards being taxed for welfare spending, feeling close to identity group, and whether subject remembered group identity), two returned a difference in distribution significantly different from zero: No appeal vs Poor MJ - Income appeal treatment in age and no appeal vs Rich MI-Group appeal treatment in feeling close to identity group. 
Table B.3: Treatment balance: summary statistics of exit-survey responses

\begin{tabular}{|c|c|c|c|c|c|c|c|c|c|c|}
\hline \multirow[b]{2}{*}{ Variable } & \multicolumn{5}{|c|}{ No appeal } & \multicolumn{5}{|c|}{ Group appeal } \\
\hline & Obs & Mean & Std. dev & Min & Max & Obs & Mean & Std. dev & Min & Max \\
\hline Age & 68 & 24.47 & 5.06 & 18 & 50 & 79 & 24.25 & 5.47 & 18 & 49 \\
\hline German & 63 & .59 & .50 & 0 & 1 & 76 & .71 & .46 & 0 & 1 \\
\hline Welfare & 68 & 2.26 & .89 & 1 & 5 & 80 & 2.58 & 1.13 & 1 & 5 \\
\hline Taxed for education & 68 & .59 & .50 & 0 & 1 & 80 & .59 & .50 & 0 & 1 \\
\hline Taxed for welfare & 68 & .18 & .38 & 0 & 1 & 80 & .11 & .32 & 0 & 1 \\
\hline Feel close to group & 68 & 5.54 & 2.95 & 0 & 10 & 80 & 5.41 & 3.06 & 0 & 10 \\
\hline Klee & 70 & .50 & .50 & 0 & 1 & 80 & .50 & .50 & 0 & 1 \\
\hline \multirow[t]{2}{*}{ Remember group ID } & 29 & 1 & 0 & 1 & 1 & 0 & . & . & . & . \\
\hline & \multicolumn{5}{|c|}{ Income appeal } & \multicolumn{5}{|c|}{ Poor $M J-$ No appeal } \\
\hline Variable & Obs & Mean & Std. dev & Min & Max & Obs & Mean & Std. dev & Min & Max \\
\hline Age & 37 & 25.76 & 5.43 & 20 & 45 & 41 & 25.39 & 4.86 & 18 & 43 \\
\hline German & 31 & .65 & .49 & 0 & 1 & 40 & .68 & .47 & 0 & 1 \\
\hline Welfare & 38 & 2.29 & .98 & 1 & 5 & 45 & 2.58 & 1.18 & 1 & 5 \\
\hline Taxed for education & 40 & .68 & .47 & 0 & 1 & 45 & .71 & .46 & 0 & 1 \\
\hline Taxed for welfare & 40 & .18 & .39 & 0 & 1 & 45 & .13 & .34 & 0 & 1 \\
\hline Feel close to group & 40 & 5.95 & 2.84 & 0 & 10 & 44 & 4.89 & 3.20 & 0 & 10 \\
\hline Klee & 40 & .50 & .51 & 0 & 1 & 45 & .49 & .51 & 0 & 1 \\
\hline \multirow[t]{2}{*}{ Remember group ID } & 40 & 1 & 0 & 1 & 1 & 45 & 1 & 0 & 1 & 1 \\
\hline & \multicolumn{5}{|c|}{ Poor $M J$ - Group appeal } & \multicolumn{5}{|c|}{ Poor $M J$ - Income appeal } \\
\hline Variable & Obs & Mean & Std. dev & Min & Max & Obs & Mean & Std. dev & Min & Max \\
\hline Age & 52 & 24.75 & 3.85 & 18 & 39 & 37 & 26.54 & 5.27 & 18 & 45 \\
\hline German & 40 & .68 & .47 & 0 & 1 & 33 & .52 & .51 & 0 & 1 \\
\hline Welfare & 53 & 2.32 & .92 & 1 & 5 & 39 & 2.54 & 1.00 & 1 & 5 \\
\hline Taxed for education & 55 & .60 & .49 & 0 & 1 & 39 & .59 & .50 & 0 & 1 \\
\hline Taxed for welfare & 55 & .22 & .42 & 0 & 1 & 39 & .18 & .39 & 0 & 1 \\
\hline Feel close to group & 54 & 6.19 & 2.51 & 0 & 10 & 40 & 5.93 & 3.08 & 0 & 10 \\
\hline Klee & 55 & .51 & .50 & 0 & 1 & 40 & .50 & .51 & 0 & 1 \\
\hline \multirow[t]{2}{*}{ Remember group ID } & 39 & 1 & 0 & 1 & 1 & 40 & 1 & 0 & 1 & 1 \\
\hline & \multicolumn{5}{|c|}{ Rich $M I$ - Group appeal } & & & & & \\
\hline Variable & Obs & Mean & Std. dev & Min & Max & & & & & \\
\hline Age & 8 & 24.38 & 2.50 & 21 & 28 & & & & & \\
\hline German & 8 & .63 & .52 & 0 & 1 & & & & & \\
\hline Welfare & 9 & 2.89 & 1.27 & 1 & 5 & & & & & \\
\hline Taxed for education & 9 & .78 & .44 & 0 & 1 & & & & & \\
\hline Taxed for welfare & 9 & .11 & .33 & 0 & 1 & & & & & \\
\hline Feel close to group & 10 & 7.70 & 2.79 & 2 & 10 & & & & & \\
\hline Klee & 10 & .50 & .53 & 0 & 1 & & & & & \\
\hline Remember group ID & 10 & 1 & 0 & 1 & 1 & & & & & \\
\hline
\end{tabular}




\section{B.4 Experimental instructions for No appeal, Group appeal, and Income appeal treatments (English translation, original in German)}

\section{Introduction}

This is an experiment on decision-making. In this experiment you will make a series of choices. At the end of the experiment, you will be paid depending on the specific choices that you made and the choices made by other participants. If you follow the instructions and make appropriate decisions, you may make up to 21 Euro. For convenience, your payoff be initially calculated in tokens and converted into Euros at the end of the experiment.

This experiment has 2 parts. Your total earnings will be the sum of your payoffs in each part plus the show-up fee of 5 Euro. We will start with a brief instruction period, followed by Part 1 of the experiment. We will then pause to receive instructions for Part 2. If you have questions during the instruction period, please raise your hand after I have completed this reading of the instructions, an experimenter will come to you and answers your questions. If you have any questions after the paid session of the experiment has begun, raise your hand, and an experimenter will come and assist you.

\section{Part 1}

\section{Assigned painter groups}

In Part 1 of the experiment, everyone will be shown five pairs of paintings by two artists, Paul Klee and Wassily Kandinsky. You will be asked to choose which painting in each pair you prefer. You will then be classified as member of the "KLEEs" (or "a KLEE" as a shorthand) or member of the "KANDINSKYs" (or "a KANDINSKY" as a shorthand) based on which artist you prefer most and informed privately about your classification. Your classification as KLEE or KANDINSKY is based on your preferences but also on how close your preferences are to the preferences of other participants' that received the same classification as yourself. Everyone's identity as a KLEE or as a KANDINSKY will stay fixed for the rest of the experiment (that is, in both Part 1 and Part 2 of the experiment). We will refer to the group of participants who share your classification as either KLEE or KANDINSKY as your painter group.

You will then be asked to identify the painter (Klee or Kandinsky) of five other paintings. For each of those paintings, you will be asked to submit two answers: your initial guess and your final answer. After submitting your initial guess, you will have an opportunity to see the initial guesses of your fellow KLEEs if you are a KLEE, or of fellow KANDINSKYs if you are a KANDINSKY, and then also an opportunity to change your answer when you are submitting your final answer.

If you are a KLEE and a half or more of KLEEs give a correct final answer then, regardless of whether your own final answer was correct or incorrect, you and each of your fellow KLEEs will receive 10 tokens. Similarly, if you are a member of the KANDINSKYs and a half or more of KANDINSKYs give a correct final answer then, regardless of your own final answer, each of the KANDINSKYs, including you, will receive 10 tokens. However, if you are a KLEE and more than a half of KLEEs give an incorrect final answer, then, regardless of whether your own final answer was correct or incorrect, you and each of the KLEEs will receive 0 tokens. And similarly, if you are a KANDINSKY and the final answers from more than a half of KANDINSKYs were incorrect, then you and each of your fellow KANDINSKYs will receive 0 tokens regardless of what answer he or a she gave personally.

In addition, if you and your fellow painter group members answer at least as many quiz ques- 
tions correctly than members of the other group, you will receive an additional payoff of 10 tokens. That is, if you are a KLEE and you and your fellow KLEEs give more correct answers than the KANDINSKYs, you receive the additional payoff. If you are a KANDINSKY and you and your fellow KANDINSKYs give more correct answers than the KLEEs, you receive the additional payoff.

We will now run Part 1 of the experiment. After Part 1 has finished, we will give you instructions for Part 2. 


\section{Part 2}

We will now move on to Part 2 of the experiment. Part 2 will consist of $\mathbf{4 0}$ different rounds.

\section{Assigned decision groups}

At the beginning of each round, you are randomly matched into groups of five participants. We will refer to those groups as your decision group. You will stay in your decision group for the duration of the experiment; that is, you will interact with the same 4 participants in all rounds of part 2 of the experiment. All participants interaction, however, will take place anonymously through a computer terminal so you do not know which participants are in your decision group.

\section{Assigned income}

At the beginning of each round, you are randomly assigned a level of income in tokens. This income determines your payoff from this part of the experiment; your payoff, however, will be mainly determined by your decisions and the decisions of other participants in your decision group. The income assigned to you is one from the following list of feasible incomes:

\section{$10,22,27,38,44,56,62,73$, or 90}

You might be assigned any of the feasible incomes and you will be assigned a new income in every round; that means, your income may or may not change from round to round and throughout the experiment, you may or may not be assigned each one of the feasible incomes at some point.

\section{Information about your decision group}

In each round, after all participants have been assigned an income, you are informed about the income and painter group membership with the KLEEs or KANDINSKYs of all participants in your decision group. Everybody, is shown a graph plotting income and associated painter group memberships on a line ranging from 0 on the left end to 100 on the right end. KLEEs are displayed with the acronym "KL" and KANDINSKYs with the acronym "KA". An exemplifying plot of an artificially created distribution of income and painter group membership is shown on page 6 (Figure 1) of these instructions.

\section{Choices within each round}

In each round, you are offered a choice between two alternatives, Alternative $A$ and Alternative $B$. Whichever alternative is chosen by a majority of participants in your decision group becomes the winning alternative of your decisions group.

\section{Payoffs}

How much money you receive for participating in this experiment will depend on the choices that you and the choices that other participants make during the experiment. For convenience, your payoff for each round will be initially calculated in tokens and reported to you at the end of each round. At the end of the session, the sum of payoffs you will have received for each round will be converted into Euro at the rate of

\section{0 tokens $=10$ Euro}

You will receive the higher round payoff out of two randomly chosen rounds plus the payoff from part 1 and the show-up fee of 5 Euro.

In each round your payoff is computed as 


$$
\text { round payoff }=\text { decision payoff }+ \text { identity payoff }
$$

Your decision payoff depends on your income and the winning alternative in your decision group. The following table displays your decision payoff given your income and the winning alternative.

Table B.4: Decision payoff given income and winning alternative

\begin{tabular}{r|cc} 
& \multicolumn{2}{|c}{ Decision payoff given } \\
Your income & Alternative A wins & Alternative B wins \\
& & \\
\hline $\mathbf{1 0}$ & 30 & 10 \\
$\mathbf{2 2}$ & 36 & 22 \\
$\mathbf{2 7}$ & 38.5 & 27 \\
$\mathbf{3 8}$ & 44 & 38 \\
$\mathbf{4 4}$ & 47 & 44 \\
$\mathbf{5 6}$ & 53 & 56 \\
$\mathbf{6 2}$ & 56 & 62 \\
$\mathbf{7 3}$ & 61.5 & 73 \\
$\mathbf{9 0}$ & 70 & 90 \\
& & \\
\hline
\end{tabular}

For example, say your income is 27 and Alternative $A$ is the winning alternative; in this case your decision payoff would be 38.5 tokens. In case Alternative B wins, however, your decision payoff would be 27 tokens.

Your identity payoff depends on whether you and the KLEES, if you are a KLEE, or you and the KANDINSKYs, if you are KANDINSKY, represent a majority among participants that voted for winning alternative in your decision group. You and the KLEEs represent a majority if more KLEEs than KANDINSKYs voted for the winning alternative. You and the KANDINSKYs represent a majority if more KANDINSKYs than KLEEs voted for the winning alternative.

Should you and the KLEEs, if you are a KLEE, or you and the KANDINSKYs, if you are a KANDINSKY, represent a majority among participants that voted for the winning alternative in your decision group, your identity payoff would be

\section{0 tokens}

Should you and the KLEEs, if you are KLEE, or you and the KANDINSKYs, if you are a KANDINSKY, not represent a majority among participants that voted for the winning alternative in your decision group, your identity payoff would be 0 tokens. Should the number of KLEEs and KANDINSKYs that voted for the winning alternative be equal, all participants in your decision group would receive 5 tokens. 
Suppose for example that you are a KLEE and there are three KLEEs in your decision group including yourself; suppose further that all participants in your decision group, including yourself, vote for Alternative A. Alternative A would be the winning alternative and you and the KLEEs would represent a majority among participants in your decision group that voted for the winning alternative. Your identity payoff would be 10 tokens.

Your payoff in this round would be the sum of your decision payoff and your identity payoff. In the aforementioned example with your income of 27, with Alternative $A$ as winning alternative, and with you and the KLEEs representing a majority of votes for the winning alternative, your payoff would be

$$
38.5+10=48.5 \text { Tokens }
$$

Should, however, the 2 KANDINSKYs and one KLEE in our decision group vote for Alternative $B$, Alternative $B$ would be the winning alternative and you and the KLEEs would not any longer represent a majority of votes for the winning alternative in your decision group; now, your payoff would be

\section{Tokens}

Again, your total earnings from this experiment are the higher round payoff out of two randomly chosen rounds plus the payoff from part 1 and the show-up fee of 5 Euro. 


\section{B.5 Income distributions}

Table B.5: Income distributions by round

\begin{tabular}{|c|c|c|c|c|c|c|c|c|c|c|c|c|}
\hline \multirow{3}{*}{$\begin{array}{l}\text { Round } \\
1\end{array}$} & \multicolumn{5}{|c|}{$\begin{array}{c}\text { Rich } M J \\
\text { treatments }\end{array}$} & \multirow{3}{*}{$\begin{array}{l}\text { Group heterogeneity } \\
\text { treatment }\end{array}$} & \multicolumn{5}{|c|}{$\begin{array}{c}\text { Poor MJ } \\
\text { treatments }\end{array}$} & \multirow{3}{*}{$\begin{array}{c}\text { Group heterogeneity } \\
\text { treatment }\end{array}$} \\
\hline & \multicolumn{3}{|c|}{$M J$} & \multicolumn{2}{|c|}{$M I$} & & \multirow[b]{2}{*}{78} & \multicolumn{2}{|c|}{$M J$} & \multicolumn{2}{|c|}{$M I$} & \\
\hline & 22 & 62 & 73 & 27 & 38 & & & 38 & 27 & 73 & 62 & \\
\hline 2 & 27 & 56 & 73 & 22 & 44 & Medium heterogeneity & 73 & 44 & 27 & 78 & 56 & Medium heterogeneity \\
\hline 3 & 27 & 56 & 73 & 22 & 44 & Medium heterogeneity & 73 & 44 & 27 & 78 & 56 & Medium heterogeneity \\
\hline 4 & 44 & 62 & 73 & 27 & 38 & Low heterogeneity & 56 & 38 & 27 & 73 & 62 & Low heterogeneity \\
\hline 5 & 44 & 62 & 73 & 27 & 38 & Low heterogeneity & 56 & 38 & 27 & 73 & 62 & Low heterogeneity \\
\hline 6 & 22 & 62 & 73 & 27 & 38 & Medium heterogeneity & 78 & 38 & 27 & 73 & 62 & Medium heterogeneity \\
\hline 7 & 22 & 62 & 73 & 27 & 38 & Medium heterogeneity & 78 & 38 & 27 & 73 & 62 & Medium heterogeneity \\
\hline 8 & 22 & 62 & 73 & 27 & 38 & Medium heterogeneity & 78 & 38 & 27 & 73 & 62 & Medium heterogeneity \\
\hline 9 & 22 & 62 & 73 & 27 & 38 & Medium heterogeneity & 56 & 38 & 27 & 73 & 62 & Low heterogeneity \\
\hline 10 & 27 & 56 & 73 & 22 & 44 & Medium heterogeneity & 56 & 44 & 27 & 73 & 62 & Low heterogeneity \\
\hline 11 & 27 & 56 & 73 & 22 & 44 & Medium & 73 & 44 & 27 & 78 & 56 & Medium heterogeneity \\
\hline 12 & 27 & 56 & 73 & 22 & 44 & Medium & 73 & 44 & 27 & 78 & 56 & Medium \\
\hline 13 & 44 & 62 & 73 & 27 & 38 & Low hete & 56 & 38 & 27 & 73 & 62 & ogeneity \\
\hline 14 & 27 & 56 & 73 & 22 & 44 & Medium heter & 73 & 44 & 27 & 78 & 56 & Medium heterogeneity \\
\hline $1:$ & 22 & 62 & 73 & 27 & 38 & Medium h & 78 & 38 & 27 & 73 & 62 & Medium heterogeneity \\
\hline 16 & 22 & 62 & 73 & 27 & 38 & eity & 56 & 44 & 27 & 73 & 62 & \\
\hline 17 & 22 & 62 & 73 & 27 & 38 & neity & 78 & 38 & 27 & 73 & 62 & Medium heterogeneity \\
\hline 18 & 22 & 62 & 73 & 27 & 38 & geneity & 78 & 38 & 27 & 73 & 62 & Medium heterogeneity \\
\hline 19 & 27 & 56 & 73 & 22 & 44 & eity & 73 & 44 & 27 & 78 & 56 & Medium hete \\
\hline 20 & 27 & 56 & 73 & 22 & 44 & eity & 73 & 44 & 27 & 78 & 5 & Medi \\
\hline 21 & 27 & 56 & 73 & 22 & 44 & eity & 56 & 44 & 27 & 73 & 62 & Low heterogeneity \\
\hline 22 & 22 & 62 & 73 & 27 & 38 & & 78 & 38 & 27 & 73 & 62 & Medium heterogeneity \\
\hline 23 & 27 & 56 & 73 & 22 & 44 & Medium heterogeneity & 73 & 44 & 27 & 78 & 56 & Medium heterogeneity \\
\hline 24 & 27 & 56 & 7 & 22 & 44 & seneity & 73 & 44 & 27 & 78 & 56 & Medium het \\
\hline 25 & 22 & 62 & 73 & 27 & 38 & eity & 78 & 38 & 27 & 73 & 62 & Med \\
\hline 26 & 27 & 56 & 73 & 22 & 44 & Medium heterogeneity & 73 & 44 & 27 & 78 & 56 & Medium heterogeneity \\
\hline 27 & 44 & 62 & 73 & 27 & 38 & Low heterogeneity & 56 & 38 & 27 & 73 & 62 & Low heterogeneity \\
\hline 28 & 22 & 62 & 73 & 27 & 38 & Medium heterogeneity & 78 & 38 & 27 & 73 & 6 & Medium heterogeneity \\
\hline 29 & 44 & 62 & 73 & 27 & 38 & Low heterogeneity & 56 & 44 & 27 & 73 & & Low heterogeneity \\
\hline 30 & 44 & 62 & 73 & 27 & 38 & Low heterogeneity & 56 & 44 & 27 & 73 & 62 & Low heterogeneity \\
\hline 31 & 27 & 56 & 73 & 22 & 44 & Medium heterogeneity & 73 & 44 & 27 & 78 & 56 & Medium heterogeneity \\
\hline 32 & 22 & 62 & 73 & 27 & 38 & Medium heterogeneity & 78 & 38 & 27 & 73 & 62 & Medium heterogeneity \\
\hline 33 & 22 & 62 & 73 & 27 & 38 & Medium het & 78 & 38 & 27 & 73 & 62 & Medium heterogeneity \\
\hline 34 & 27 & 56 & 73 & 22 & 44 & Medium heterogeneity & 73 & 44 & 27 & 78 & 56 & Medium heterogeneity \\
\hline 35 & 44 & 62 & 73 & 27 & 38 & Low heterogeneity & 56 & 38 & 27 & 73 & 62 & Low heterogeneity \\
\hline 36 & 44 & 62 & 73 & 27 & 38 & Low heterogeneity & 56 & 38 & 27 & 73 & 62 & Low heterogeneity \\
\hline 37 & 10 & 56 & 90 & 22 & 44 & & 90 & 44 & 10 & 78 & 56 & High heterogeneity \\
\hline 38 & 10 & 56 & 90 & 22 & 44 & & 90 & 44 & 10 & 78 & 56 & High heterogeneity \\
\hline 39 & 10 & 56 & 90 & 22 & 44 & High heterogeneity & 90 & 44 & 10 & 78 & 56 & High heterogeneity \\
\hline 40 & 10 & 56 & 90 & 22 & 44 & High heterogeneity & 90 & 44 & 10 & 78 & 56 & High heterogeneity \\
\hline
\end{tabular}




\section{B.6 Screen shot}

Figure B.5: Screen shot of subjects' decision between Alternative A and Alternative B (German original). English Translation: Round 1: You are a Klee / Your income is 27./ Here are the incomes of all participants of your society: / Please make your choice between alternative A and alternative B now. / You chose alternative A. / Please press continue to proceed.

Runde 1: Sie sind ein KLEE

Ihr Einkommen ist 27.

Hier sind die Einkommen aller Teilnehmer in Ihrer Entscheidungsgruppe:

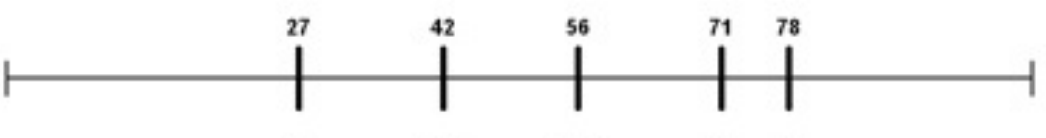

KL $\quad$ KA $\quad$ KA $\quad K L \quad K L$

Bittle tretten Sie nun thre Wahl zwischen Alternative A und Alternative B.
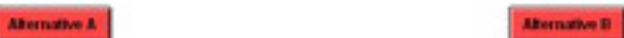

Sie haben sich für Alternative A entschieden.

Bitte drücken Sie Weiter um tortzufahren. 


\section{Statistical appendix}

\section{C.1 Summary statistics}

Table C.6: Relative frequency of strategy profiles by group heterogeneity and appeal treatments

\begin{tabular}{rrrrr} 
& & \multicolumn{3}{c}{ Group heterogeneity treatments } \\
\cline { 5 - 5 } Variable & Appeal treatments & Low & Medium & High \\
\hline & & & & \\
$\mathrm{P}$ wins, all vote $\mathrm{P}$ & No appeal & 0.01 & 0.21 & 0.16 \\
$(P$-equilibrium $)$ & Group appeal & 0.03 & 0.19 & 0.25 \\
& Income appeal & 0.03 & 0.15 & 0.31 \\
$\mathrm{P}$ wins, $M J$ or $M I$ & No appeal & 0.28 & 0.53 & 0.68 \\
split & Group appeal & 0.24 & 0.51 & 0.61 \\
& Income appeal & 0.47 & 0.46 & 0.69 \\
$\mathrm{R}$ wins, $M J$ or $M I$ & No appeal & 0.29 & 0.16 & 0.14 \\
split & Group appeal & 0.21 & 0.11 & 0.02 \\
& Income appeal & 0.12 & 0.19 & 0.00 \\
$\mathrm{R}$ wins, $M J$ votes for & No appeal & 0.42 & 0.10 & 0.02 \\
$R$ and $M I$ votes for $P$ & Group appeal & 0.52 & 0.19 & 0.12 \\
& Income appeal & 0.38 & 0.20 & 0.00
\end{tabular}


Table C.7: Summary statistics of main variables by income and appeal treatments. Statistics are pooled across all levels of group heterogeneity, subjects, and rounds within one treatment.

\begin{tabular}{|c|c|c|c|c|c|c|c|c|}
\hline & \multirow[b]{2}{*}{ Variable } & \multicolumn{3}{|c|}{ Main treatments } & \multicolumn{4}{|c|}{ Supplemental treatments } \\
\hline & & $\begin{array}{l}\text { No appeal } \\
\text { Mean (SD) }\end{array}$ & $\begin{array}{l}\text { Group appeal } \\
\text { Mean (SD) }\end{array}$ & $\begin{array}{l}\text { Income appeal } \\
\text { Mean (SD) }\end{array}$ & $\begin{array}{l}\text { Poor } M J- \\
\text { No Appeal } \\
\text { Mean (SD) } \\
\end{array}$ & $\begin{array}{c}\text { Poor } M J- \\
\text { Group Appeal }\end{array}$ & $\begin{array}{c}\text { Poor } M J- \\
\text { Income Appeal } \\
\text { Mean }(\mathrm{SD}) \\
\end{array}$ & $\begin{array}{c}\text { Rich } M I- \\
\text { Group appeal } \\
\text { Mean (SD) } \\
\end{array}$ \\
\hline \multirow{5}{*}{ vote $R$} & All & $.38(.49)$ & $.38(.49)$ & $.39(.49)$ & $.71(.45)$ & $.61(.49)$ & $.68(.47)$ & $.92(.27)$ \\
\hline & Very poor & $.23(.42)$ & $.24(.43)$ & $.24(.43)$ & $.47(.50)$ & $.44(.50)$ & $.48(.50)$ & $.75(.44)$ \\
\hline & Moderately poor & $.30(.46)$ & $.20(.40)$ & $.24(.43)$ & $.56(.50)$ & $.47(.50)$ & $.48(.50)$ & $.90(.31)$ \\
\hline & Moderately rich & $.54(.50)$ & $.58(.49)$ & $.61(.49)$ & $.80(.40)$ & $.66(.47)$ & $.77 .(42)$ & $.93(.26)$ \\
\hline & Very rich & $.59(.49)$ & $.65(.48)$ & $.63(.48)$ & $.86(.34)$ & $.76(.43)$ & $.84(.38)$ & $.98(.16)$ \\
\hline$R$ wins election & All & $.34(.48)$ & $.37(.48)$ & $.38(.48)$ & $.83(.38)$ & $.58(.49)$ & $.74(.44)$ & $1.0(0.0)$ \\
\hline income & All & $45(20)$ & $45(20)$ & $45(20)$ & $55(19)$ & $54(19)$ & $54(20)$ & $60(17)$ \\
\hline \multirow{2}{*}{\multicolumn{2}{|c|}{$\begin{array}{l}\text { Number of Observations } \\
\text { Number of Subjects }\end{array}$}} & 2800 & 3200 & 1600 & 1800 & 2200 & 1600 & 300 \\
\hline & & 70 & 80 & 40 & 45 & 55 & 40 & 10 \\
\hline
\end{tabular}




\section{C.2 Additional statistical analysis}

Table C.8: Multi-level random effects regression of indicator for strategy profile $(R, R, R ; P, P)$ being played and of indicator for strategy profile $(P, P, P ; P, P), P$-equilibrium, being played on group heterogeneity treatment, appeal treatment, interaction of those treatments, and round of play including random intercepts for societies.

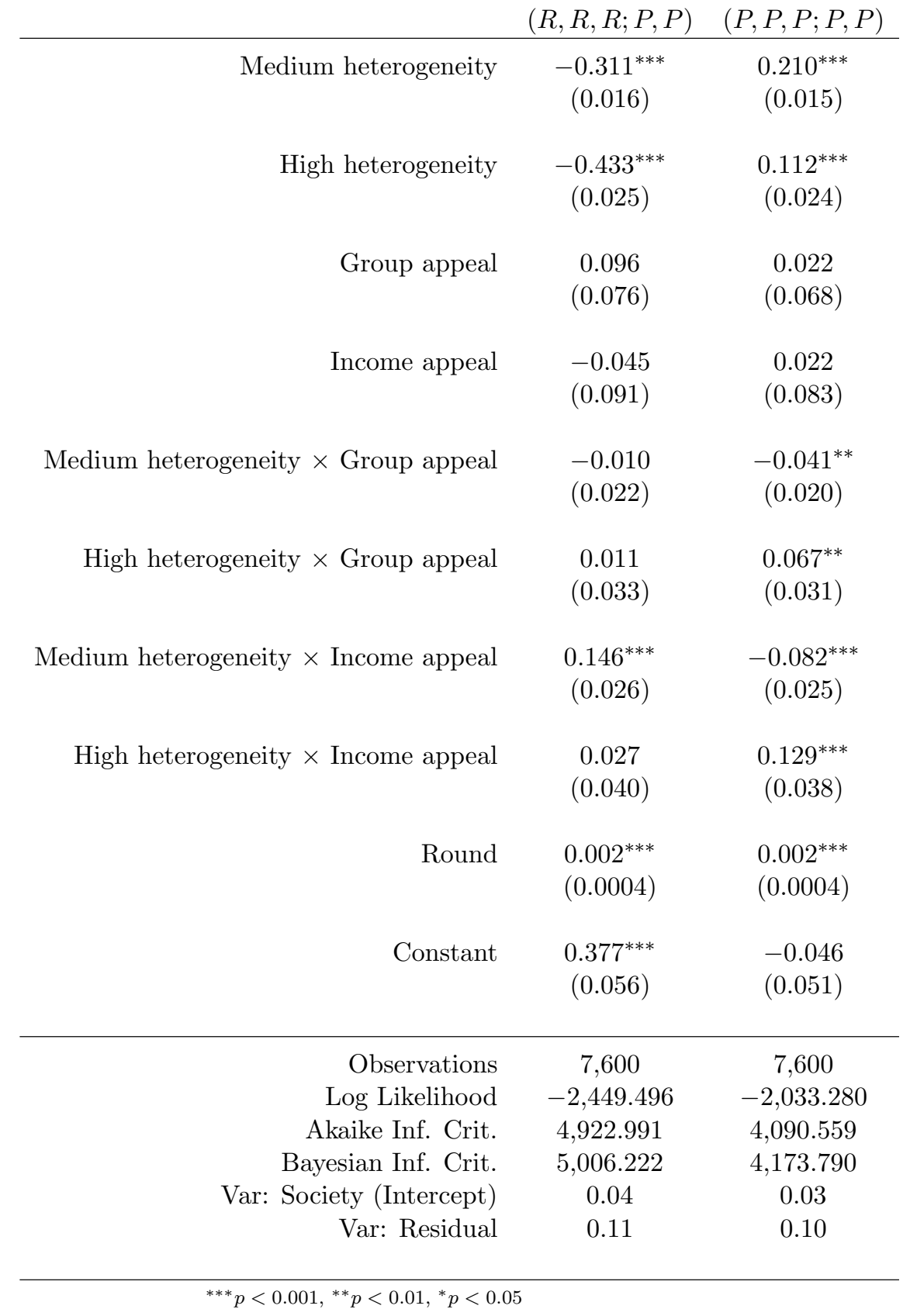


Figure C.6: Distribution of relative frequency of strategy profiles by group heterogeneity and appeal treatments in first third (round 1-12), second third (round 13-24), and final third (round 25-36) of the experiment. Observations on the high group heterogeneity treatment (round 37-40) are omitted.
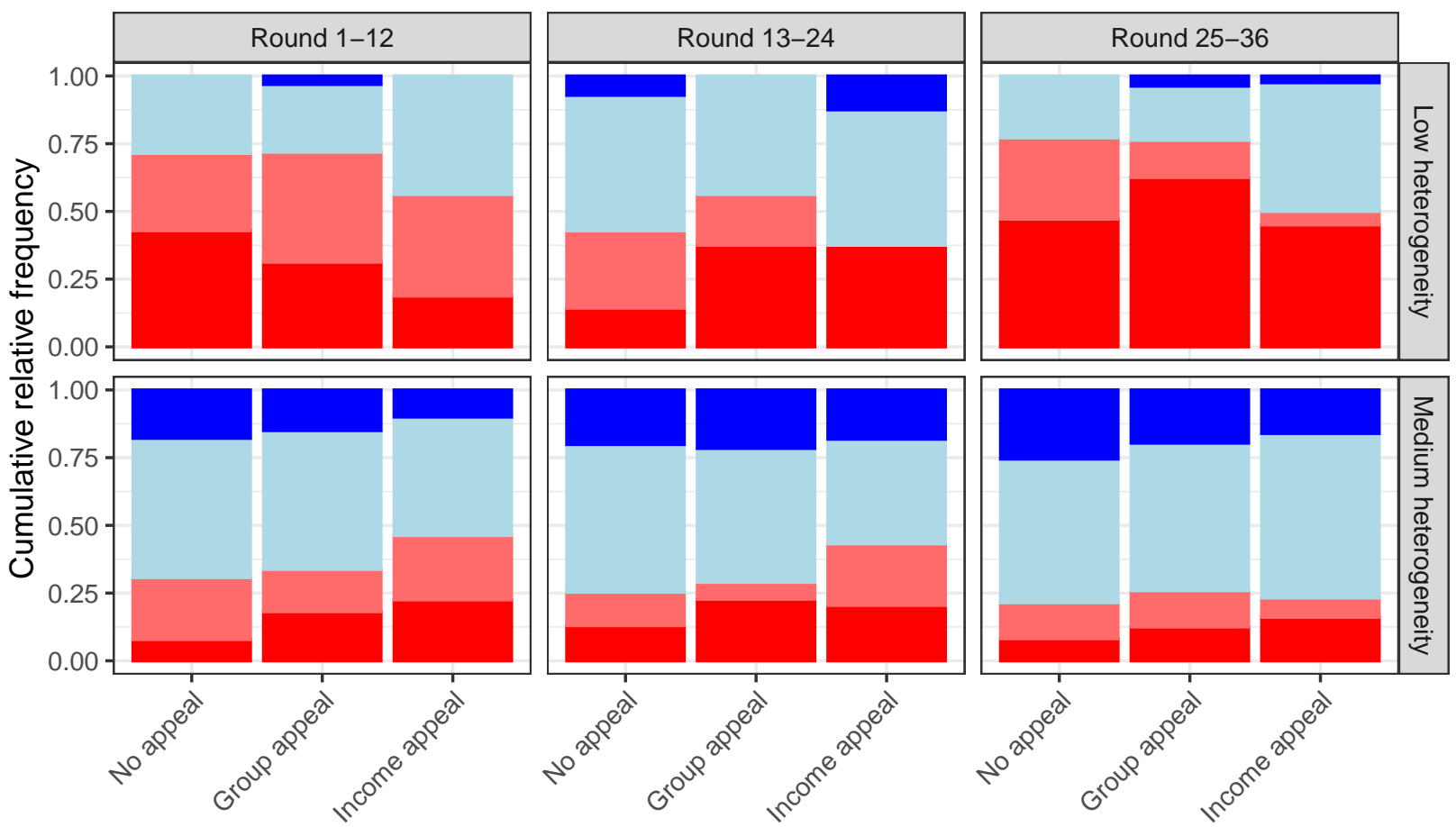

Redistributive candidate $\mathrm{P}$ wins, all vote $\mathrm{P}$ Redistributive candidate $\mathrm{P}$ wins, MJ or MI split

\section{References}

Fischbacher, Urs. 2007. "z-Tree: Zurich Toolbox for Ready-made Economic Experiments." Experimental Economic 10(2):171-178.

Greiner, Ben. 2015. "Subject pool recruitment procedures: organizing experiments with ORSEE." Journal of the Economic Science Association pp. 1-12. 